\title{
Stochastic geometry modeling and analysis of cognitive heterogeneous cellular networks
}

\author{
Fereidoun H Panahi ${ }^{*}$ and Tomoaki Ohtsuki
}

\begin{abstract}
In this paper, we present a cognitive radio (CR)-based statistical framework for a two-tier heterogeneous cellular network (femto-macro network) to model the outage probability at any arbitrary secondary (femto) and primary (macro) user. A system model based on stochastic geometry (utilizing the spatial Poisson point process (PPP) theory) is applied to model the random locations and network topology of both secondary and primary users. A considerable performance improvement can be generally achieved by mitigating interference in result of applying the CR idea over the above model. Novel closed-form expressions are derived for the downlink outage probability of any typical femto and macro user considering the Rayleigh fading for the desired and interfering links. We also study some important design factors which their role and importance in the determination of outage and interference cannot be ignored. We conduct simulations to validate our analytical results and evaluate the proposed schemes in terms of outage probability for different values of signal-to-interference-plus-noise ratio (SINR) target.
\end{abstract}

Keywords: Heterogeneous cellular network; Cognitive radio; Outage probability

\section{Introduction}

The best solution to the spectrum saturation and bandwidth availability problems in multi-tier cellular networks is to adopt technologies that make the most efficient use of existing spectrum through frequency reuse schemes $[1,2]$. In universal frequency reuse scheme, the existing spectrum can be aggressively and effectively reused by all of the coexisting network tiers. This will lead to higher spatial spectrum utilization and network usage capacity at the expense of an increased possibility of interference among network tiers and of a reduced quality of service (QoS). In multi-tier cellular networks, interference is increasingly becoming a major performancelimiting factor, and hence, interference modeling, coordination, and avoidance are the primary focus of interest for both the industry and academic communities. Applying the cognitive radio $(\mathrm{CR})$ technology in multi-tier cellular networks to be aware of and adapt to communication environments, some of the above challenges can be tackled. In fact, CR is the key enabling technology for interference management and avoidance in multi-tier cellular networks $[2,3]$. On the other hand, the aggregate interference

\footnotetext{
* Correspondence: fereidoun@ohtsuki.ics.keio.ac.jp

Graduate School of Science and Technology, Keio University, 3-14-1 Hiyoshi,
} Kouhoku-ku, Yokohama 223-8522, Japan environment is more complicated to model, and evaluating the performance of communication techniques in the presence of heterogeneous interference is challenging. For interference characterization, if the base stations (BSs) of the cellular network follow a regular grid (e.g., the traditional hexagonal grid model), then the SINR characterization will be either intractable [3,4] or inaccurate due to unrealistic assumptions [5]. Moreover, as urban areas are built out, the BS infrastructure is becoming less like points on a hexagonal lattice and more random. Hence, the use of a hexagonal grid to model the BS locations is violated and is considered too idealized [6]. Furthermore, according to $[3,4,6]$ for snapshots of a cellular network at different locations, the positions of the BSs with respect to each other follow random patterns due to the size and unpredictability of the BSs in these kind of networks. Therefore, the need for a powerful mathematical and statistical tool for modeling, analysis, and design of wireless networks with random topologies is quite obvious.

A new modeling approach called 'stochastic geometry' has been recently applied to the analysis of multi-tier cellular networks due to its ability to capture the topological randomness in the network and its aim at deriving accurate and tractable expressions for outage probability $[3,6]$. 
Stochastic geometry stems from applied probability and has a wide range of applications in the analysis and design of wireless networks in particular for modeling and analyzing systems with random channel access (e.g., ALOHA $[7,8]$ and carrier sensing multiple access (CSMA) [9]), single- and multi-tier cellular networks [6], and networks with cognitive abilities $[7,10]$. Multi-tier cellular networks have been investigated from different perspectives such as power control [11,12], spectrum allocation [13,14], and exploiting CR techniques [15,16], and recently, many works have been done based on the similar concepts to adopt and extend the stochastic geometric approach to different network models and scenarios (see [17-20]). This paper discusses this new theoretical model to provide a better understanding of the heterogeneous cellular networks of tomorrow and their challenges (interference modeling, coordination, and avoidance) that must be tackled in order for these networks to reach their potential. We focus on a two-tier femto-macro network where low-power and small-coverage local nodes (femto nodes) are distributed in the coverage of macro nodes. We provide an insight into the role of CR in interference mitigation in two-tier heterogeneous networks. We derive closed-form expressions for the outage probability of any typical femto and macro user in the network. We also study the effect of several important design factors which play vital roles in the determination of outage and interference.

Our main contributions in this work which is an extension of [21] are therefore the following: (1) We analyze the Laplace transforms of all four types of aggregate interference between macro and CR femto networks (including the interference between macro nodes among themselves and femto nodes among themselves, the cross-interference from femto to macro network and vice versa) in perfect and imperfect spectrum sensing CR-based femto networks, considering simultaneously the Poisson point process (PPP) model, and some important design factors (such as spectrum access probability) which can play a major role in determining interference and outage. (2) This article provides an insight into the role of $\mathrm{CR}$ in interference mitigation in orthogonal frequency-division multiple-access (OFDMA) two-tier heterogeneous networks. (3) Closedform expressions are derived for the outage probability of any typical femto and macro user considering the Rayleigh fading assumption for the desired and interfering links with the possibility of using the CR ability for the femto network. It should be noted that in most of the available studies in this area, none of the network tiers is equipped with the CR capability; they are mostly based on the existence of only one macro-BS (along with the macro users and the femto network); and the effect of considering multiple macro-BSs is ignored in the analysis of outage probability. Authors in [22-25] have considered the twotier heterogeneous networks imposing the $\mathrm{CR}$ ability to the femto tier. Different from [22], in our work, we consider both the perfect and imperfect sensing scenarios for the CR femto-BSs, however authors in [22] ignore the effect of sensing errors on the opportunistic channel access probability and consequently the outage probability of each tier. On the other hand, in our work, the mathematical demonstration of the obtained expressions (channel access probability and outage probability expressions) is quite different from the mentioned works.

\section{Downlink system model}

\subsection{Model description}

We consider infinite spatially collocated macro-BS and femto-BS node heterogeneous networks (see Figure 1). It is assumed that the spatial distribution of the nodes is captured using two collocated and independent homogenous Poisson point processes (HPPPs) [6,7] i.e., $\Phi_{M}$ and $\Phi_{F}$ with intensities $\lambda_{M}$ and $\lambda_{F}$ respectively. In other words, the locations of the macro-BS nodes constitute an HPPP $\Phi_{M}$, where $\lambda_{M}$ is the average number of the macro-BS nodes per unit area. Similar statement can be made for the HPPP formed by the femto-BS nodes $\Phi_{F}$ with intensity $\lambda_{F}$. According to Superposition Theorem [26], the overall node process over the network formed by both the macro-BS and femto-BS nodes also is an HPPP with intensity $\lambda\left(\lambda=\lambda_{F}+\lambda_{M}\right)$. Furthermore, the macro and femto users are scattered about the plane according to some independent PPPs with different densities compared to $\lambda_{M}$ and $\lambda_{F}$ respectively. However, our interference analysis is fundamentally concerned with the distribution of the transmitters (BSs).

Since femto-BSs are installed and maintained by the paying home users for better indoor performance, they are only accessible by their own mobile subscribers (femto users) (known as closed-access policy). On the other hand, macro-BSs can be accessed only by unauthorized users (macro users). In practice, macro network is deployed usually without awareness of the distributed femto network. To this end, wireless operators can consider giving priority to the macro users, and the femto network has to be self-optimized to mitigate its interference to the macro users. Motivated by this insight, the macro-BSs (along with the macro users) and the CR-enabled femto-BSs (along with the femto users) are analogous to primary and secondary systems in the CR model, respectively.

\subsection{System structure}

In OFDMA, the spectrum is orthogonally divided into time-frequency resource blocks (RBs), which increases flexibility in resource allocation, thereby allowing high spectral efficiency. As shown in Figure 2, we consider a spectrum of $N$ RBs, out of which $M(M \leq N)$ random RBs are idle or unoccupied by the macro users (primary system). With the CR capability, a femto-BS could actively 


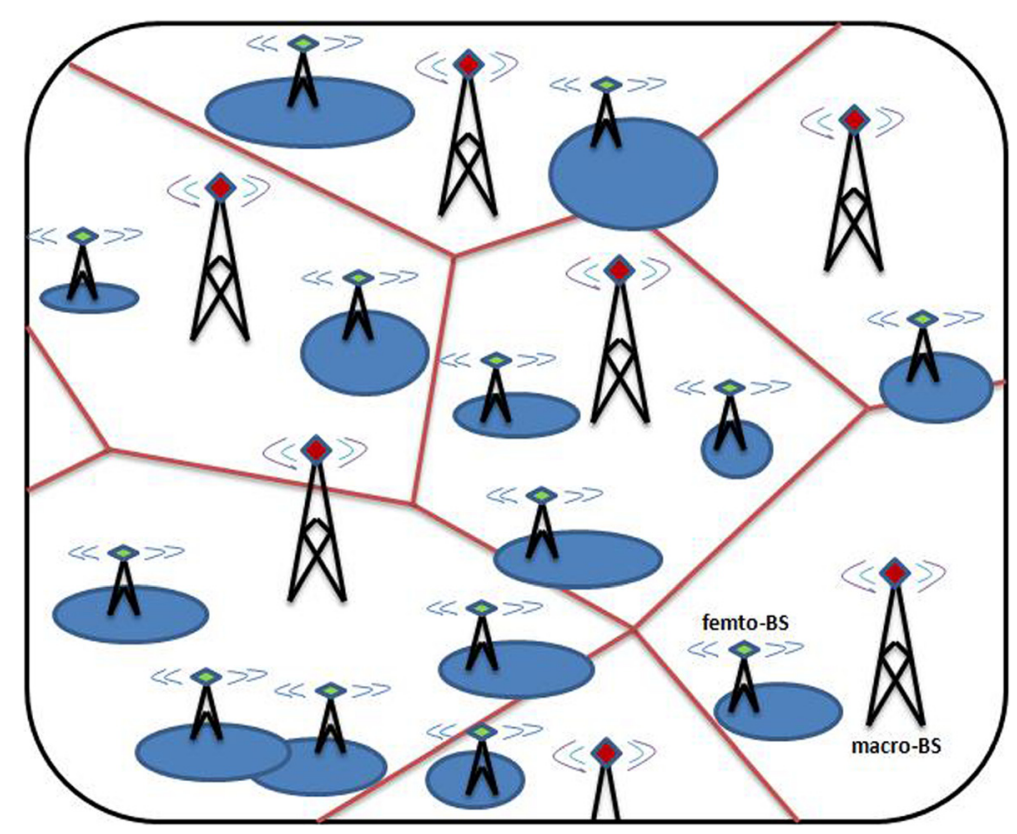

Figure 1 The heterogeneous model (femto and macro BSs).

acquire knowledge about its environment and access to the RBs without the aid of a macrocell in a decentralized fashion (clearly, no synchronization between the macro and femto network is needed any more) and automatically prevent disturbing the macro users [2].

1) As shown in Figure 2, each femto-BS's transmission strategy is divided into consecutive slots, each having a duration of $T$. Each slot is divided into two consecutive stages, i.e., sensing and data transmission, with durations of $T_{S}$ and $T_{D}$, respectively. Each femto-BS periodically senses the spectrum to identify which RBs are occupied by the macro network.
Indeed, each femto-BS accomplishes sensing one RB in one unit slot $T_{S R B}$ within $T_{S}$. Each femto-BS senses $N_{s}$ RBs in sequence which is randomly selected from the $N$ available RBs, and detects its idle RB set. Clearly, the time required for sensing the $N_{s}$ RBs is $T_{S}=T_{S R B} N_{S}$. Note that the femto-BSs cannot perform data transmission within the sensing time $T_{S}$. We assume that all femto-BSs are perfectly synchronized and have the same time as the sensing time. Methods for implementing a perfect synchronization among the femto-BSs are outside the scope of this paper; however, a set of possible candidates exist, including GPS synchronization, the wired backhaul (IEEE 1588),

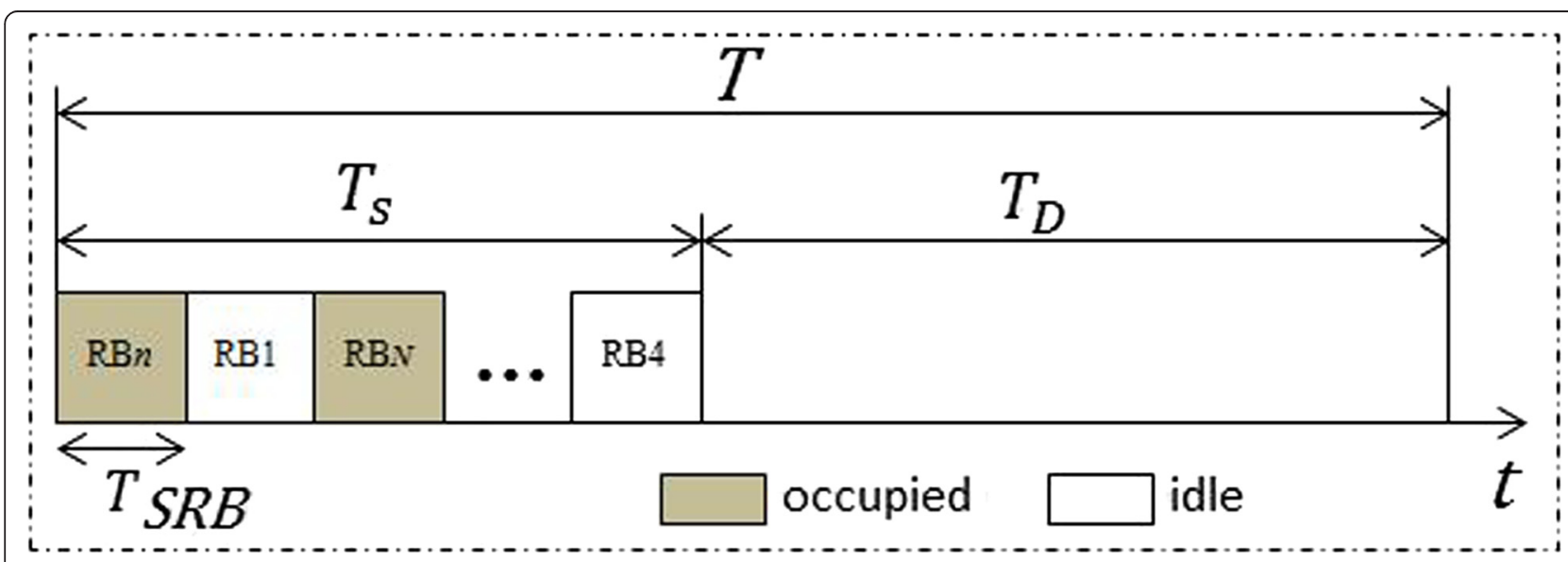

Figure 2 The CR femto-BS's transmission strategy in one time slot. 
and leveraging synchronization signals broadcasted by the femto-BSs [27].

2) Each femto-BS senses the received interference power on each RB within the sensing duration:

- If the received interference power on an $\mathrm{RB}$ at a typical femto-BS exceeds a certain threshold, the $\mathrm{RB}$ is identified as being occupied by one or more macro nodes but not by the femto network since all the femto-BSs have the same sensing time (It should be noted that if an RB is identified as being occupied at a typical femto-BS, it does not necessarily mean that it is also seen as an occupied RB at the other femto-BSs, as this status determination process depends only on the received interference power level on the $R B$ at each individual femto-BS).

- Otherwise, the RB is unoccupied by the macro network.

3) In the data transmission time $\left(T_{D}\right)$, each femto-BS only allocates an unoccupied RB sensed in the sensing time to its user (by only utilizing these unoccupied RBs, cross-tier interference can be consequently avoided). Since the determination of each individual RB status as busy/idle is subject to (occasional) error, determined by the probability of (correct) detection of the presence of PUs' signals $P_{d}$ and probability of false alarm $P_{f}$ (probability of falsely declaring an idle RB as busy), we study the effect of both the ideal detection, i.e., $P_{d}=1$ and $P_{f}=0$, and the cases involving imperfect sensing (see $[28,29]$ ), i.e., $P_{d} \neq 1$ and $P_{f} \neq 0$ on the outage probabilities of femto and macro users.

In each realization of the point process, each macro and femto user communicates only with its nearest macro-BS and femto-BS, respectively. As shown in Figure 3, the macro users' exclusion regions with radius $D$ are used to guarantee that the femto-BSs will, on average, not generate an aggregate interference leading to the outage of macro (primary) users. We assume that the macro users can be localized, e.g., based on pilot signals or transmitted acknowledgements. Therefore, the femto-BSs inside the macro users' exclusion regions may be able to detect the macro signals and cease their transmissions. As shown in the figure, for example, those femto-BSs located in the tagged macro user's exclusion region are not allowed to transmit data whether they pick the same RB as the tagged macro user or a different one. It should be noted that the tagged macro user is

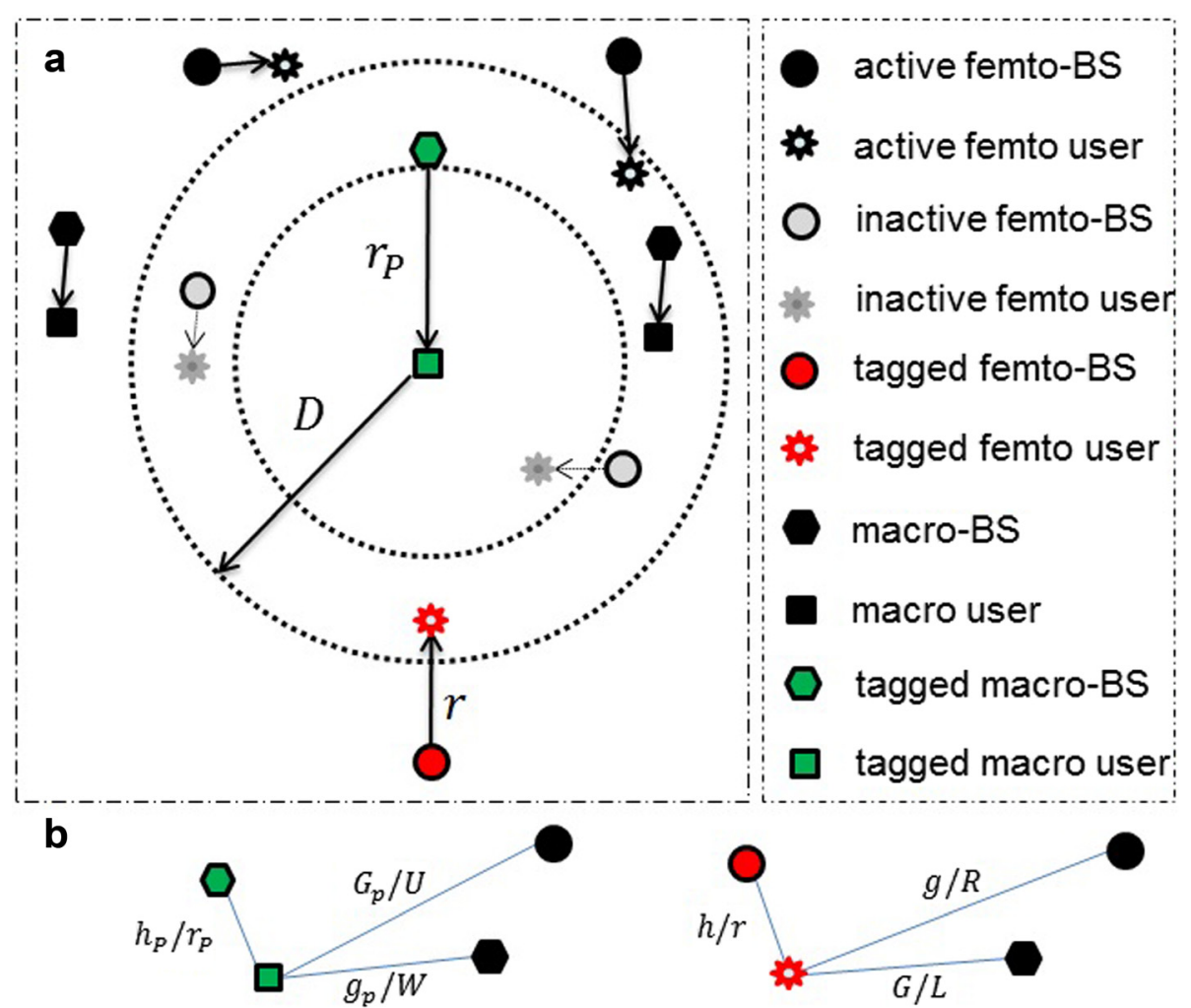

Figure 3 Bipolar network model and communication links. (a) The bipolar network model. (b) The link gains/distances. 
not disturbed by the femto-BSs transmitting on different RBs (from that of the tagged macro) even if they are inside its exclusion region. However, we deactivate them to protect the tagged macro user from any harmful interference as a result of possible errors in their sensing and location detection processes (we consider this law throughout the paper even in the case of the perfect sensing scenario). For instance, if a femto-BS, located at a very close distance from the tagged macro user, wrongly (the cases involving imperfect sensing) picks the occupied RB by the tagged macro user, it will cause a severe interference to the tagged macro user if it is not able to perfectly detect the location of the tagged macro user (e.g., because of the hidden node problem in CR systems [30]) to cease its transmission.

\section{Stochastic geometry-based network configuration}

\subsection{Femto outage probability formulation}

We derive the probability of outage for a typical femto user $\left(p_{\mathrm{OF}}\right)$ in a downlink heterogeneous cellular network defined as the probability that a randomly chosen femto user cannot achieve a target SINR $\theta$ (or equivalently as the average fraction of femto users who do not achieve a $\operatorname{target} \operatorname{SINR} \theta$, i.e., the averaged outage probability of all femto users) considering a collocated spectrum sensing CR-based femto network and macro-BSs as follows [6]:

$$
p_{\mathrm{OF}}=1-P[\operatorname{SINR}>\theta]
$$

In fact, the outage probability evaluates the CDF of SINR over the entire network. The experienced SINR by a typical femto user is calculated as

$$
\operatorname{SINR}=\frac{P_{F} h r^{-\alpha}}{\sigma^{2}+I_{\mathrm{FB}}+I_{\mathrm{MB}}}
$$

where $P_{F}$ is the transmission power from the nearest femto-BS (tagged femto-BS) located in the random distance $r$ from its tagged femto user (we assume that the tagged femto user under consideration is located at the origin), and $\alpha$ is the path-loss exponent. $I_{\mathrm{FB}}$ and $I_{\mathrm{MB}}$ are the aggregate interference power at the origin from the other femto-BSs and macro-BSs, respectively, and $\sigma^{2}$ is the noise power. It should be noted that the transmission power values of all the femto-BSs in the network are kept constant, i.e., $P_{F}$.

Let $h \sim \exp (\mu)$ (with mean $1 / \mu$ ) be a random variable accounting for the random channel gain of the link between the tagged femto user and its corresponding femto-BS, and then we have

$$
\begin{aligned}
P[\operatorname{SINR} & >\theta] \\
& =P\left[\frac{P_{F} h r^{-\alpha}}{\sigma^{2}+I_{\mathrm{FB}}+I_{\mathrm{MB}}}>\theta\right] \\
& \ddot{=} P\left[h>\left(\sigma^{2}+I_{\mathrm{FB}}+I_{\mathrm{MB}}\right) \frac{\theta r^{\alpha}}{P_{F}}\right] \\
& \stackrel{=}{=} \mathrm{E}_{I_{\mathrm{FB}}, I_{\mathrm{MB}}}\left[\int_{\left(\sigma^{2}+I_{\mathrm{FB}}+I_{\mathrm{MB}} \frac{\theta \sigma_{F}}{P_{F}}\right.}^{\infty}[\mu \exp (-\mu \mathrm{x})] \mathrm{dx}\right] \\
& =\mathrm{E}_{I_{\mathrm{FB}}, I_{\mathrm{MB}}}\left[\exp \left[-\mu\left(\sigma^{2}+I_{\mathrm{FB}}+I_{\mathrm{MB}}\right) \frac{\theta r^{\alpha}}{P_{F}}\right]\right]
\end{aligned}
$$

where $(.$.$) is derived by rearranging the terms in SINR;$ $(\cdots)$ is derived by noting that $h$ is an exponential random variable; and $\mathrm{E}_{I_{\mathrm{EB}}, I_{\mathrm{MB}}}[\cdot]$ is the expectation operator with respect to the joint distribution of the random variables $I_{\mathrm{FB}}$ and $I_{\mathrm{MB}}$.

Note that due to the assumption of independent PPPs for the femto and macro networks, the aggregate interference received from the femto-BSs is independent of the aggregate interference received from the macro-BSs [10], therefore, we can write

$$
\begin{aligned}
P[\text { SINR }>\theta] & =e^{-\mu \frac{\theta r^{\alpha}}{P_{F}} \sigma^{2}} \cdot \mathrm{E}_{\mathrm{IF}_{\mathrm{FB}}}\left[e^{-\mu \frac{\theta r^{\alpha}}{P_{F}} I_{\mathrm{FB}}}\right] \cdot \mathrm{E}_{I_{\mathrm{MB}}}\left[e^{\left.-\mu \frac{\theta r^{\alpha}}{P_{F}} I_{\mathrm{MB}}\right]}\right. \\
& =e^{-\mu \frac{\theta r^{\alpha}}{P_{F}} \sigma^{2}} \cdot \mathcal{L}_{I_{\mathrm{FB}}}\left(\mu \frac{\theta r^{\alpha}}{P_{F}}\right) \cdot \mathcal{L}_{I_{\mathrm{MB}}}\left(\mu \frac{\theta r^{\alpha}}{P_{F}}\right)
\end{aligned}
$$

where $\mathcal{L}_{I_{\mathrm{FB}}}(s)$ and $\mathcal{L}_{I_{\mathrm{MB}}}(s)$ are the Laplace transform of random variables $I_{\mathrm{FB}}$ and $I_{\mathrm{MB}}$ evaluated at $s\left(s=\mu \frac{\theta r}{P_{F}}\right)$, respectively. Thus, the probability of outage averaged over the plane is derived as

$$
\begin{gathered}
p_{\mathrm{OF}}=1-\mathrm{E}_{r}[P[\operatorname{SINR}>\theta]] \\
p_{\mathrm{OF}}=1-\int_{0}^{\infty}[P[\operatorname{SINR}>\theta]] \cdot f_{r}(r) \mathrm{dr}
\end{gathered}
$$

where $f_{r}(r)=e^{-\lambda_{F} \pi r^{2}} 2 \pi \lambda_{F} r$ (as mentioned before, $r$ is the random distance between the tagged femto user and its corresponding femto-BS) is the probability density function (pdf) of $r$ [6]. Then, we have

$$
\begin{gathered}
p_{\mathrm{OF}}=1-\int_{0}^{\infty} e^{-\pi \lambda_{F} r^{2}} \cdot e^{-\mu \frac{\theta r_{F}^{\alpha}}{P_{F}} \sigma^{2}} \cdot \mathcal{L}_{I_{\mathrm{FB}}}\left(\mu \frac{\theta r^{\alpha}}{P_{F}}\right) \\
. \mathcal{L}_{I_{\mathrm{MB}}}\left(\mu \frac{\theta r^{\alpha}}{P_{F}}\right) \cdot 2 \pi r \lambda_{F} \mathrm{dr}
\end{gathered}
$$




\subsubsection{Scenario I}

Ideal detection $\left(P_{d}=1\right.$ and $\left.P_{f}=0\right)$ :

Each secondary node (femto-BS) has perfect knowledge of each primary (macro-BS) signaling. In other words, sensing at each femto-BS is done perfectly. Therefore, an RB occupied by a macro-BS is not chosen for data transmission by the nearby femto-BSs. Under this condition, the tagged femto user, during the data transmission time, does not experience any interference from the macro-BSs since it always communicates with its corresponding femto-BS on an idle RB. In fact, we assume that the received interference power from the macro network under this scenario can be neglected if it is measured to be less than a specified threshold (if we do not neglect the received interference power under the explained condition, then the outage probability formulations will be the same as in Scenario II except for the $\mathrm{RB}$ selection probability expressions, $p_{\mathrm{RB}}$, as we explain later. Similar arguments can be made for the outage probability of the macro tier as discussed in the next subsections).

Therefore, under this assumption, $\mathrm{E}_{I_{\mathrm{MB}}}\left[e^{-\mu \theta_{P_{F}, \alpha}} I_{\mathrm{MB}}\right]=1$ and $\mathcal{L}_{I_{\mathrm{MB}}}\left(\mu \frac{\theta r^{\alpha}}{P_{F}}\right)=1$, consequently. Then (3) is rewritten as follows

$$
p_{\mathrm{OF}}=1-\int_{0}^{\infty} e^{-\pi \lambda_{F} r^{2}} \cdot e^{-\mu \frac{\theta \theta^{\alpha}}{P_{F}} \sigma^{2}} \cdot \mathcal{L}_{I_{\mathrm{FB}}}\left(\mu \frac{\theta r^{\alpha}}{P_{F}}\right) \cdot 2 \pi r \lambda_{F} \mathrm{~d} r
$$

The Laplace transform of the aggregate interference from all the active femto-BSs except the tagged femto$\mathrm{BS}$ denoted by $f b s_{O}$ is given as follows (Note: some of the femto-BSs located in the macro users' exclusion regions with radius $D$ are deactivated, therefore, $\lambda_{F}^{\prime} \leq \lambda_{F}$ (see Figure 3))

$$
\begin{aligned}
\mathcal{L}_{I_{\mathrm{FB}}}(s) & =\mathrm{E}_{I_{\mathrm{FB}}}\left[\exp \left(-s I_{\mathrm{FB}}\right)\right] \\
& =\mathrm{E}_{\Phi_{F}}, g_{i}\left[\exp \left(-s \sum_{i \in \Phi_{F} \backslash\left\{f b s_{0}\right\}} P_{F} g_{i} R_{i}^{-\alpha}\right)\right] \\
& =\mathrm{E}_{\Phi_{F}}\left[\prod_{i \in \Phi_{F} \backslash\left\{f b s_{0}\right\}} \mathrm{E}_{g_{i}}\left[\exp \left(-s P_{F} g_{i} R_{i}^{-\alpha}\right)\right]\right]
\end{aligned}
$$

Where $R_{i}$ is the distance of the $i$ th interferer from the tagged femto receiver captured by the point process $\Phi_{F}$ The interference channel gains $g_{i}$ are assumed to be mutually independent and have identical pdfs. Each of the active interfering femto-BSs transmits with the same power $P_{F}$. Using the definition of the Generating functional [6] for the Poisson point process (PPP), which states for some function $f(x)$ that $\mathrm{E}\left[\prod_{x \in \Phi} f(x)\right]=\exp \left(-\int_{\mathbb{R}^{d}}(1-f(x)) \lambda \mathrm{dx}\right)$,

be rewritten as

$$
\mathcal{L}_{I_{\mathrm{FB}}}(s)=\exp \left\{-\mathrm{E}_{g}\left[\int_{r}^{\infty}\left(1-\exp \left(-s P_{F} g R^{-\alpha}\right)\right) \lambda_{I}(R) \mathrm{d} R\right]\right\}
$$

where we flipped the order of integration and expectation. Since the closest interfering femto-BS is at least at distance $r$ from the tagged user, the integration limits are from $r$ to $\infty$. In other words, interference is encountered from all the active femto-BSs located in the area $\mathbb{R}^{d} \backslash b(0, r)$ (where $b(x, y)$ is ball of radius $y$ centered at point $x$ ). However, not all the femto-BSs will contribute towards the aggregate interference, i.e., only those active femto-BSs located outside the mentioned ball which at minimum satisfy all of the following conditions qualify as potential contributors.

Before explaining these conditions ((a) and (b)), it is useful to translate the point process into polar coordinates. Therefore, according to $[7,26]$, the intensity of the HPPP $\Phi_{F}$ is shown as

$$
\lambda_{I}(R)=\lambda_{F}^{\prime} d R^{d-1} b_{d}
$$

where $R$ is the distance between an arbitrary femto-BS and the tagged femto receiver. $b_{d}$ is the volume of a unit sphere in $\mathbb{R}^{d}\left(b_{d}=\frac{\sqrt{\pi^{d}}}{\Gamma\left(1++^{d} / 2\right)}, \Gamma(x)=\int_{0}^{\infty} t^{x-1} e^{-t} \mathrm{~d} t\right.$ denotes the standard Gamma function).

(a) Satisfying the aforementioned condition, any active femto-BS contributes towards the interference at the tagged femto receiver, if it picks the same RB as the tagged femto-BS to communicate with its user. We show the probability of picking a same RB from a pool of all RBs as $p_{\mathrm{RB}}$ (the calculation of $p_{\mathrm{RB}}$ for this case is derived in Section 4, Scenario I, Case 1).

(b) We assume that the CR femto-BSs employ a slotted ALOHA MAC (medium access control) protocol to schedule their transmission. Therefore, they only transmit with probability $p_{\mathrm{tx}}$ in the current time slot and defer the transmission with probability $1-p_{\mathrm{tx}}$.

Applying both conditions (a) and (b) (which will result in reducing the number of interferers and hence outage probability at the tagged femto user), $\Phi_{F}$ is thinned using two independent thinning. Indeed, first, $\Phi_{F}$ is reconstructed by $p_{\mathrm{RB}}$ thinning, where each point of $\Phi_{F}$ is retained with probability $p_{\mathrm{RB}}$ (to accommodate the 
condition (a)), and then it is thinned again by applying the other independent thinning (to accommodate the condition (b)). Thus, the intensity of the process (the number of the interfering $\mathrm{CR}$ femto-BSs) becomes

$$
\lambda_{I}(R)=\lambda_{F}^{\prime} d R^{d-1} b_{d} p_{\mathrm{RB}} p_{\mathrm{tx}}
$$

Now, (6) is rewritten as

$$
\exp \left\{-\mathrm{E}_{g}\left[\int_{r}^{\infty}\left(1-\exp \left(-s P_{F} g R^{-\alpha}\right)\right) \lambda_{F}^{\prime} b_{d} p_{\mathrm{RB}} p_{\mathrm{tx}} d R^{d-1} \mathrm{~d} R\right]\right\} .
$$

By using the change of variables $R^{d} \rightarrow x$ and then $x^{\frac{\alpha}{d}} \rightarrow y$, and doing some simple calculations, the above expression is simplified as:

$$
\mathcal{L}_{I_{\mathrm{FB}}}(s)=e^{r^{d} b_{d} p_{\mathrm{RB}} p_{\mathrm{tx}} \lambda_{F}^{\prime}-\frac{d}{\alpha} b_{d} p_{\mathrm{RB}} p_{\mathrm{tx}} \lambda_{F}^{\prime}(\mu \theta)^{\frac{d}{\alpha} r^{d}} M(\theta, \alpha)}
$$

where

$$
M(\theta, \alpha)=\mathrm{E}\left[(g)^{\frac{d}{\alpha}}\left(\Gamma\left(-\frac{d}{\alpha}, \mu \theta g\right)-\Gamma\left(-\frac{d}{\alpha}\right)\right)\right]
$$

Note that $\Gamma(a, x)=\int_{x}^{\infty} t^{a-1} e^{-t} \mathrm{~d} t$ indicates the incomplete gamma function. From (4) and (9), taking $d=2$, then replacing $r^{2}$ with $z$, we get the final formula for the outage probability of the tagged femto user as follows

$$
\begin{aligned}
p_{\mathrm{OF}}= & 1-\int_{0}^{\infty} e^{\pi z\left(\lambda_{F}^{\prime} p_{\mathrm{RB}} p_{\mathrm{xx}}-\lambda_{F}\right)-\frac{\mu \theta}{T_{F}} \sigma^{2} z^{\frac{\alpha}{2}}-\frac{2}{\alpha} \pi p_{\mathrm{RB}} p_{\mathrm{tx}} \lambda_{F}^{\prime}(\mu \theta)^{\frac{2}{z}} z M(\theta, \alpha)} \\
& \pi \lambda_{F} \mathrm{~d} z
\end{aligned}
$$

in which $M(\theta, \alpha)=\mathrm{E}\left[(g)^{\frac{2}{\alpha}}\left(\Gamma\left(-\frac{2}{\alpha}, \mu \theta g\right)-\Gamma\left(-\frac{2}{\alpha}\right)\right)\right]$. According to [13], the downlink femtocell networks are assumed to be interference-limited, i.e., the noise can be neglected as the interference dominates the whole performances of the system $\sigma^{2} \rightarrow 0$.

Thus, (11) reduces to (12) as follows $(\alpha=4)$

$$
p_{\mathrm{OF}}=1-\frac{\lambda_{F}}{\left(\lambda_{F}-\lambda_{F}^{\prime} p_{\mathrm{RB}} p_{\mathrm{tx}}\right)+\frac{\lambda_{F}^{\prime}\left(p_{\mathrm{RB}} p_{\mathrm{tx}}\right)}{2} \sqrt{\mu \theta} M(\theta, 4)} .
$$

Similar with the desired link, we consider the Rayleigh fading model for the femto interfering links as well (Rayleigh fading links with equal parameter $\mu$ ). Then following the derivation of $M(\theta, \alpha)$ in Appendix I, $p_{\mathrm{OF}}$ is re-expressed as follows

$$
\begin{aligned}
& p_{\mathrm{OF}} \\
& =1-\frac{\lambda_{F}}{\left(\lambda_{F}-\lambda_{F}^{\prime} p_{\mathrm{RB}} p_{\mathrm{tx}}\right)+\sqrt{\pi} \lambda_{F}^{\prime}\left(p_{\mathrm{RB}} p_{\mathrm{tx}}\right) \mu\left[\sum_{k=0}^{\infty} \frac{(\mu \theta)^{k}}{\Gamma\left(k+\frac{1}{2}\right)(\mu+\mu \theta)^{k+1}} \Gamma(1+k)\right]} .
\end{aligned}
$$

\subsubsection{Scenario II} Imperfect detection $\left(P_{d} \neq 1\right.$ and $\left.P_{f} \neq 0\right)$ :

In this scenario, each secondary node (femto-BS) has imperfect knowledge of each primary (macro-BS) signaling. In other words, sensing at each femto-BS is done imperfectly. Therefore, an occupied RB by a macro-BS may also be wrongly considered idle by the femto-BSs, causing collision between the two networks. Two cases can take place under the imperfect sensing scenario:

Case 1. The tagged femto-BS transmits data on an idle RB (for this case, the outage probability formulations can be considered the same as in the perfect sensing scenario except for the calculation of $p_{\mathrm{RB}}$ (see Section 4, Scenario II, Case 1))

Case 2. The tagged femto-BS transmits on an occupied $\mathrm{RB}$ (outage probability formulation in this case is explained as follows and the calculation of $p_{\mathrm{RB}}$ is presented in Section 4, Scenario II, Case 2)

In Case 2, the tagged femto user can experience interference from both the active femto-BSs (which pick the same occupied RB as the tagged femto-BS) and macroBSs. Indeed, the $\mathcal{L}_{I_{\mathrm{MB}}}\left(\mu \frac{\theta r^{\alpha}}{P_{F}}\right)$ (the Laplace transform of the aggregate interference from the macro-BSs) in (3) is not ignored. The Laplace transform of the aggregate interference power generated by the macro-BSs at the tagged femto user is given by

$$
\begin{aligned}
& \mathcal{L}_{I_{\mathrm{MB}}}(s)=\mathrm{E}_{I_{\mathrm{MB}}}\left[\exp \left(-s I_{\mathrm{MB}}\right)\right] \\
& =\mathrm{E}_{\Phi_{M}, G_{i}}\left[\exp \left(-s \sum_{i \in \Phi_{M}} P_{P} G_{i} L_{i}^{-\alpha}\right)\right] \\
& =\mathrm{E}_{\Phi_{M}}\left[\prod_{i \in \Phi_{M}} \mathrm{E}_{G_{i}}\left[\exp \left(-s P_{P} G_{i} L_{i}^{-\alpha}\right)\right]\right]
\end{aligned}
$$

where $L_{i}$ is the distance of the $i$ th interfering macro-BS from the tagged femto receiver captured by the point process $\Phi_{M}$. The interference channel gains $G_{i}$ are assumed to be mutually independent and have identical pdfs. Each of the active interfering macro-BSs transmits with the same power $P_{P}$. 
Again, using the definition of the generating functional for the PPP, we can write

$$
\mathcal{L}_{I_{\mathrm{MB}}}(s)=\exp \left\{-\mathrm{E}_{G}\left[\int_{0}^{\infty}\left(1-\exp \left(-s P_{P} G L^{-\alpha}\right)\right) \lambda_{I}(L) \mathrm{d} L\right]\right\} .
$$

The interference is encountered from all the macroBSs located in the area $\mathbb{R}^{d} \backslash b(0,0)$. It should be noted that not all the macro-BSs in $\mathbb{R}^{d}$ will contribute towards the aggregate interference, i.e., only those macro-BSs which are transmitting on the same $\mathrm{RB}$ as the tagged femto user qualify as potential interferers. The intensity of the HPPP $\Phi_{M}$ process can be therefore written as follows

$$
\lambda_{I}(L)=\lambda_{M}^{\prime} d L^{d-1} b_{d}
$$

where $L$ is the distance between an arbitrary macro-BS and the tagged femto receiver and $\lambda_{M}^{\prime}$ is the intensity of those macro-BSs transmitting on the same $\mathrm{RB}$ as the tagged femto user at a time.

Now, (15) is rewritten as

$$
\exp \left\{-\mathrm{E}_{G}\left[\int_{0}^{\infty}\left(1-\exp \left(-s P_{P} G L^{-\alpha}\right)\right) \lambda_{M}^{\prime} L^{d-1} b_{d} d L^{d-1} \mathrm{~d} L\right]\right\}
$$

By using the change of variables and doing some simple calculations, $\mathcal{L}_{I_{\mathrm{MB}}}(s)$ is obtained as follows [26]

$$
\mathcal{L}_{I_{\mathrm{MB}}}(s)=e^{-b_{d} \lambda_{M}^{\prime} \Gamma\left(1-\frac{d}{\alpha}\right)\left(\frac{P_{p} \mu \theta}{P_{F}}\right)^{\frac{d}{\alpha}} r^{d} \mathrm{E}\left[(G)^{\frac{d}{\alpha}}\right]} .
$$

From (3), (9), and (17), taking $d=2$, then replacing $r^{2}$ with $z$, and assuming $\sigma^{2} \rightarrow 0$, we get the final formula as follows

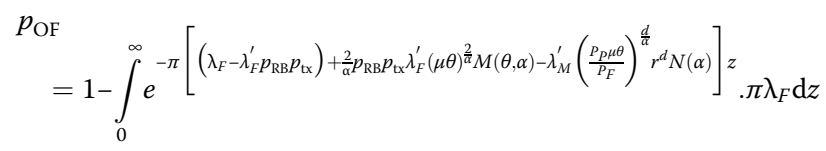

in which $M(\theta, \alpha)=\mathrm{E}\left[(g)^{\frac{2}{\alpha}}\left(\Gamma\left(-\frac{2}{\alpha}, \mu \theta g\right)-\Gamma\left(-\frac{2}{\alpha}\right)\right)\right]$ and $N(\alpha)=\Gamma\left(1-\frac{d}{\alpha}\right) \mathrm{E}\left[(G)^{\frac{d}{\alpha}}\right]$.

Finally, the closed-form expression for the outage probability of the tagged femto user, under the imperfect sensing scenario for all CR femto-BSs, is obtained as follows $(\alpha=4)$

$$
\begin{aligned}
& p_{\mathrm{OF}} \\
& =1-\frac{\lambda_{F}}{\left(\lambda_{F}-\lambda_{F}^{\prime} p_{\mathrm{RB}} p_{\mathrm{tx}}\right)+\frac{\lambda_{F}^{\prime}\left(p_{\mathrm{RB}} p_{\mathrm{tx}}\right)}{2} \sqrt{\mu \theta} M(\theta, 4)+\lambda_{M}^{\prime} N(4) \sqrt{\frac{P_{P} \mu \theta}{P_{F}}}} .
\end{aligned}
$$

Similar with the desired link, we consider the Rayleigh fading model for the femto interfering links as well as the macro interfering links (Rayleigh fading links with equal parameter $\mu$ for the femto interfering links and $\mu_{p}$ for the macro interfering links). Following the derivation of $M(\theta, \alpha)$ in Appendix I, and after the simplification of $N(\alpha)$ using the definition of expectation and the standard gamma function, $p_{\mathrm{OF}}$ is re-expressed as follows $\alpha=4$

$$
\begin{aligned}
& p_{\mathrm{OF}} \\
& =1-\frac{\lambda_{F}}{\left(\lambda_{F}-\lambda_{F}^{\prime} p_{\mathrm{RB}} p_{\mathrm{tx}}\right)+\sqrt{\pi} \lambda_{F}^{\prime}\left(p_{\mathrm{RB}} p_{\mathrm{tx}}\right) \mu\left[\sum_{k=0}^{\infty} \frac{(\mu \theta)^{k}}{\Gamma\left(k+\frac{1}{2}\right)(\mu+\mu \theta)^{k+1}} \Gamma(1+k)\right]+\frac{\pi \lambda_{M}^{\prime}}{2 \sqrt{\mu_{p}}} \sqrt{\frac{P_{P} \mu \theta}{P_{F}}}} .
\end{aligned}
$$

\subsection{Macro outage probability formulation}

We derive the outage probability for a typical macro user $\left(p_{\mathrm{OM}}\right)$ in a downlink heterogeneous cellular network defined as the probability that a randomly chosen macro user cannot achieve a target SINR $\gamma$ (or equivalently as the average fraction of macro users who do not achieve a target SINR $\gamma$, i.e., the averaged outage probability of all macro users) considering a collocated spectrum sensing CR-based femto network and macroBSs as follows:

$$
p_{\mathrm{OM}}=1-P[\operatorname{SINR}>\gamma]
$$

The experienced SINR by a typical macro user is calculated as

$$
\mathrm{SINR}=\frac{P_{P} h_{p} r_{p}^{-\alpha}}{\sigma^{2}+I_{\mathrm{MB}}+I_{\mathrm{FB}}}
$$

where $P_{P}$ is the transmission power from the nearest macro-BS located in the random distance $r_{p}$ from its tagged macro user located at the origin and $\alpha$ is the path-loss exponent. $I_{\mathrm{FB}}$ and $I_{\mathrm{MB}}$ are the aggregate interference power to the tagged macro user (located at the origin) from the surrounding femto-BSs and macro-BSs, respectively, and $\sigma^{2}$ is the noise power. It should be noted that the transmission power values of all the macro-BSs in the network are kept constant, i.e., $P_{P}$.

Let $h_{p} \sim \exp \left(\mu_{p}\right)$ (with mean $1 / \mu_{p}$ ) be a random variable accounting for the random channel gain of the link between the tagged macro user and its corresponding macro-BS, then the similar approach to obtain the outage probability of the tagged femto user is used for the 
outage probability calculation of the tagged macro user. Therefore, from (21) and (22), we have

$$
\begin{aligned}
p_{\mathrm{OM}}= & 1-\int_{0}^{\infty} e^{-\pi \lambda_{M} r_{p}^{2}} \cdot e^{-\mu_{p} r_{p} p_{p}^{\alpha} \sigma^{2}} \cdot \mathcal{L}_{I_{\mathrm{FB}}}\left(\mu_{p} \frac{\gamma r_{p}^{\alpha}}{P_{P}}\right) \\
& \mathcal{L}_{I_{\mathrm{MB}}}\left(\mu_{p} \frac{\gamma r_{p}^{\alpha}}{P_{P}}\right) \cdot 2 \pi r_{p} \lambda_{M} \mathrm{~d} r_{p}
\end{aligned}
$$

where $\mathcal{L}_{I_{\mathrm{FB}}}(s)$ and $\mathcal{L}_{I_{\mathrm{MB}}}(s)$ are the Laplace transform of random variables $I_{\mathrm{FB}}$ and $I_{\mathrm{MB}}$ evaluated at $s\left(s=\mu_{p} \frac{\gamma r_{p}^{\alpha}}{P_{P}}\right)$, respectively.

\subsubsection{Scenario I}

Ideal detection $\left(P_{d}=1\right.$ and $\left.P_{f}=0\right)$ :

Each secondary node (femto-BS) has perfect knowledge of each primary (macro-BS) signaling. In other words, sensing at each femto-BS is done perfectly. Therefore, an RB occupied by a macro-BS is not chosen for data transmission by any nearby femto-BS. Under this condition, the tagged macro user, during the data transmission time, does not experience any interference (or a negligible interference) from the surrounding femto-BSs; however, those macro-BSs operating on the same $\mathrm{RB}$ as the tagged macro user and located in the area $\mathbb{R}^{d} \backslash b\left(0, r_{p}\right)$ make interference to the tagged macro user. Therefore, (23) becomes

$$
p_{\mathrm{OM}}=1-\int_{0}^{\infty} e^{-\pi \lambda_{M} r_{p}^{2}} \cdot e^{-\mu_{p} \frac{\gamma r_{p}^{\alpha}}{P_{P}} \sigma^{2}} \cdot \mathcal{L}_{I_{\mathrm{MB}}}\left(\mu_{p} \frac{\gamma r_{p}^{\alpha}}{P_{P}}\right) \cdot 2 \pi r_{p} \lambda_{M} \mathrm{~d} r_{p}
$$

Taking a similar approach to what we had before, $\mathcal{L}_{I_{\mathrm{MB}}}(s)$ is obtained as follows

$$
\mathcal{L}_{I_{\mathrm{MB}}}(s)=\exp \left\{-\mathrm{E}_{g_{p}}\left[\int_{r_{p}}^{\infty}\left(1-\exp \left(-s P_{P} g_{p} W^{-\alpha}\right)\right) \lambda_{I}(W) \mathrm{d} W\right]\right\}
$$

and

$$
\lambda_{I}(W)=\lambda_{M}^{\prime} d W^{d-1} b_{d}
$$

in which $W$ is the distance between an arbitrary macro-BS (captured by the point process $\Phi_{M}$ ) and the tagged macro receiver and $\lambda_{M}^{\prime}$ is the intensity of those macro-BSs transmitting on the same RB as the tagged macro user at a time. Similarly, the interference channel gains $g_{p}$ (between the interfering macroBSs and the tagged macro user) are assumed to be mutually independent and have identical pdfs. Each of the active interfering macro-BSs transmits with the same power $P_{P}$ as for the tagged macro-BS. Same as before, (25) is simplified as

$$
\mathcal{L}_{I_{\mathrm{MB}}}(s)=e^{r_{p}{ }^{d} b_{d} \lambda_{M}^{\prime}-\frac{d}{\alpha} b_{d} \lambda_{M}^{\prime}\left(\mu_{p} \gamma\right)^{\frac{d}{\alpha}} r_{p}^{d} V(\gamma, \alpha)} .
$$

From (24) and (27) and the previous assumptions, the closed-form formula is expressed as follows

$$
p_{\mathrm{OM}}=1-\frac{\lambda_{M}}{\lambda_{M}-\lambda_{M}^{\prime}+\frac{\lambda_{M}^{\prime}}{2} \sqrt{\mu_{p} \gamma} V(\gamma, 4)}
$$

in which $V(\gamma, \alpha)=\mathrm{E}\left[\left(g_{p}\right)^{\frac{2}{\alpha}}\left(\Gamma\left(-\frac{2}{\alpha}, \mu_{p} \gamma g_{p}\right)-\Gamma\left(-\frac{2}{\alpha}\right)\right)\right]$.

The Rayleigh fading model is also considered for the macro interference links (Rayleigh fading links with equal parameter $\left.\mu_{p}\right)$. The derivation of $V(\gamma, \alpha)$ is similar to the derivation of $M(\theta, \alpha)$ in Appendix I. Therefore, $p_{\mathrm{OM}}$ is re-expressed as follows $(\alpha=4)$

$$
p_{\mathrm{OM}}=1-\frac{\lambda_{M}}{\lambda_{M}-\lambda_{M}^{\prime}+\sqrt{\pi} \lambda_{M}^{\prime} \mu_{p}\left[\sum_{k=0}^{\infty} \frac{\left(\mu_{p} \gamma\right)^{k}}{\Gamma\left(k+\frac{1}{2}\right)\left(\mu_{p}+\mu_{p} \gamma\right)^{k+1}} \Gamma(1+k)\right]}
$$

\subsubsection{Scenario II}

Imperfect detection $\left(P_{d} \neq 1\right.$ and $\left.P_{f} \neq 0\right)$ :

In this scenario, each secondary node (femto-BS) has imperfect knowledge of each primary (macro-BS) signaling. In other words, sensing at each femto-BS is done imperfectly and subjected to (occasional) error. Therefore, an occupied RB by a macro-BS may also be wrongly considered idle by the femto-BSs. In this situation, the tagged macro user can experience interference on its RB, from both the femto and macro BSs. Indeed, the $\mathcal{L}_{I_{\mathrm{FB}}}\left(\mu_{p} \frac{\gamma r_{p}{ }^{\alpha}}{P_{P}}\right)$ (the Laplace transform of the aggregate interference to the tagged macro user from the surrounding femto-BSs) in (23) is not ignored. Using the same approach as described before, $\mathcal{L}_{I_{\mathrm{FB}}}(s)$ is also given by

$$
\mathcal{L}_{I_{\mathrm{FB}}}(s)=\exp \left\{-\mathrm{E}_{G_{P}}\left[\int_{K r_{p}}^{\infty}\left(1-\exp \left(-s P_{F} G_{P} U^{-a}\right)\right) \lambda_{I}(U) d U\right]\right\} .
$$

Since the closest interfering femto-BS is at least at distance $K r_{p}$ from the tagged macro user, the integration limits are from $K r_{p}$ to $\infty$. In other words, interference is encountered from all the femto-BSs located in the area $\mathbb{R}^{d} \backslash b\left(0, K r_{p}\right)$ (see Figure 3). It should be noted that not all the femto-BSs outside this ball will contribute towards the aggregate interference, i.e., only those femto-BSs which are outside the mentioned ball and at 
minimum satisfy all of the following conditions are considered as potential contributors

(c) Satisfying the above condition, any arbitrary femto-BS contributes towards the interference at the tagged macro receiver, if it wrongly picks the same $\mathrm{RB}$ as the tagged macro-BS to communicate with its user. We show the probability of picking a same RB for data transmission from a pool of all RBs as $p_{\mathrm{RB}}$ (the calculation of $p_{\mathrm{RB}}$ for this case is seen in Section 4, Scenario II, Case 2).

(d)Same as the condition (b) in subsection 3.1.1.

Applying these two independent thinning, the intensity of the process (the number of the interfering CR femtoBSs at the tagged macro user) becomes

$$
\lambda_{I}(U)=\lambda_{F}^{\prime} d U^{d-1} b_{d} p_{\mathrm{RB}} p_{\mathrm{tx}}
$$

Taking the similar approach, (30) is simplified as follows

$$
\mathcal{L}_{I_{\mathrm{FB}}}(s)=e^{K^{d} r_{p}^{d} b_{d} p_{\mathrm{RB}} p_{\mathrm{tx}} \lambda_{F}^{\prime}-\frac{d}{\alpha} b_{d} p_{\mathrm{RB}} p_{\mathrm{tx}} \lambda_{F}^{\prime}\left(\mu_{p} p_{F} \frac{p_{F} Y}{P_{P}}\right)^{\frac{d}{\alpha}} r_{p}^{d} O(\gamma, \alpha)}
$$

in which $O(\gamma, \alpha)=\mathrm{E}\left[\left(G_{p}\right)^{\frac{d}{\alpha}}\left(\Gamma\left(-\frac{d}{\alpha}, \frac{\mu_{p} \gamma P_{F} G_{p}}{K^{\alpha} P_{P}}\right)-\Gamma\left(-\frac{d}{\alpha}\right)\right)\right]$.

From (23), (27), and (32) and the previous assumptions, the closed-form expression for the outage probability of the tagged macro user is obtained as follows

$$
\begin{aligned}
& p_{\mathrm{OM}}
\end{aligned}
$$

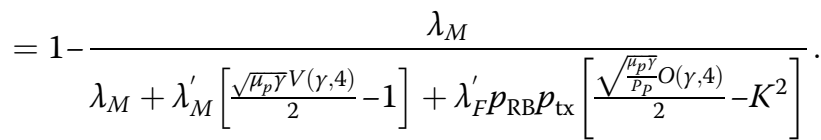

Similar with the desired link, the Rayleigh fading model for the femto interfering links (with equal parameter $\mu$ ) as well as the macro interfering links (with equal parameter $\mu_{p}$ ) is considered. Considering Appendix I and after replacing $V(\gamma, 4)$ and $O(\gamma, 4)$ with their expanded versions, $p_{\mathrm{OM}}$ can be re-expressed as follows

$$
p_{\mathrm{OM}}=1-\frac{\lambda_{M}}{\mathrm{~A}+\mathrm{B}}
$$

where

$$
\mathrm{A}=\lambda_{M}-\lambda_{M}^{\prime}+\sqrt{\pi} \lambda_{M}^{\prime} \mu_{p}\left[\sum_{k=0}^{\infty} \frac{\left(\mu_{p} \gamma\right)^{k}}{\Gamma\left(k+\frac{1}{2}\right)\left(\mu_{p}+\mu_{p} \gamma\right)^{k+1}} \Gamma(1+k)\right]
$$

and

$$
\mathrm{B}=\lambda_{F}^{\prime} p_{\mathrm{RB}} p_{\mathrm{tx}} K^{2}\left[\mu \sqrt{\frac{\pi}{P_{F}}}\left[\sum_{k=0}^{\infty} \frac{\left(\frac{\mu_{p} P_{F} P_{F}}{K^{4} P_{P}}\right)^{k}}{\Gamma\left(k+\frac{1}{2}\right)\left(\mu+\frac{\mu_{p} P_{F}}{K^{4} P_{P}} \gamma\right)^{k+1}} \Gamma(1+k)\right]-1\right]
$$

\section{Resource block selection probability ( $p_{\mathrm{RB}}$ ) calculations under perfect and imperfect sensing}

In this section, we discuss how the optimal values of the RB selection probability $\left(p_{\mathrm{RB}}\right)$ for a secondary transmitter (femto-BS) can be determined under each femto-BS's perfect and imperfect sensing scenarios.

\subsection{Scenario I}

Ideal detection for all the CR femto-BSs $\left(P_{d}=1\right.$ and $\left.P_{f}=0\right)$ $[31,32]$ :

Case 1. The tagged femto-BS assigns the $i$ th idle RB to its femto user.

$p_{\mathrm{RB}}$ : The probability that the $i$ th idle RB being selected for data transmission by any of the other active CR femto-BS [32].

$$
p_{\mathrm{RB} \mid M_{s}}=p_{\text {idle }}\left(M_{s}\right) \cdot \frac{\left(\begin{array}{c}
M-1 \\
M_{s}-1
\end{array}\right)}{\left(\begin{array}{c}
M \\
M_{s}
\end{array}\right)} \cdot\left(\frac{1}{M_{s}}\right)
$$

where

$$
p_{\text {idle }}\left(M_{s}\right)=\frac{\left(\begin{array}{c}
M \\
M_{s}
\end{array}\right)\left(\begin{array}{c}
N-M \\
N_{s}-M_{s}
\end{array}\right)}{\left(\begin{array}{c}
N \\
N_{s}
\end{array}\right)} .
$$

The first term $\left(p_{\text {idle }}\left(M_{s}\right)\right)$ indicates the probability of $M_{s}$ idle RBs sensed by a femto-BS (during the sensing time, $T_{S}$ ), the second term is the probability that the $i$ th idle RB is inside the $M_{s}$ idle RBs, and finally the third term indicates that the probability of selecting the $i$ th idle RB (out of the $M_{s}$ idle RBs) by that femto-BS is equal to $\frac{1}{M_{s}}$ (since each of the idle RBs within the $M_{s}$ idle RBs has an equal probability of being chosen). It should be noted that each CR femto-BS will fail to access when $M_{s}=$ 0 (the maximum value of $M_{s}$ is equal to $\min \left\{M, N_{s}\right\}$ ). Therefore, from (35),

$$
\begin{aligned}
p_{\mathrm{RB}}= & p_{\mathrm{RB} \mid\left(M_{s} \geq 1\right)}=\frac{1}{M} p_{\text {idle }}\left(M_{s} \geq 1\right) \\
& =\frac{1}{M}\left(1-p_{\text {idle }}\left(M_{s}=0\right)\right) .
\end{aligned}
$$

If the number of RBs sensed by a CR femto-BS (i.e., $N_{s}$ ) is more than or equal to $N-M+1$, then at least we have one idle RB within the $N_{s}$ detected RBs, i.e., $p_{\text {idle }}\left(M_{s}=0\right)=$ 0 . On the other hand, if $N_{s}$ is smaller than or equal to $N-M$, the CR femto-BS will fail to access when 
the RBs within the $N_{s}$ sensed RBs are all occupied by the macro-BSs. Therefore, we can write

$$
p_{\text {idle }}\left(M_{s}=0\right)=\left\{\begin{array}{cc}
\frac{\left(\begin{array}{c}
N-M \\
N_{s}
\end{array}\right)}{\left(\begin{array}{c}
N \\
N_{s}
\end{array}\right),} & \text { if } N_{s} \leq N-M \\
0, & \text { if } N_{s} \geq N-M+1 .
\end{array}\right.
$$

From (36) and (37), the probability that a CR femto-BS selects the $i$ th idle RB for data transmission is obtained as follows

$$
p_{\mathrm{RB}}=\left\{\begin{array}{c}
\frac{1}{M}\left(\begin{array}{c}
\left.1-\frac{\left(\begin{array}{c}
N-M \\
N_{s}
\end{array}\right)}{\left(\begin{array}{c}
N \\
N_{s}
\end{array}\right)}\right), \\
\frac{1}{M},
\end{array} \quad \text { if } N_{s} \leq N-M\right. \\
\text { if } N_{s} \geq N-M+1 .
\end{array}\right.
$$

\subsection{Scenario II}

Imperfect detection for all the CR femto-BSs $\left(P_{d} \neq 1\right.$ and $\left.P_{f} \neq 0\right)[31,32]$ :

Case 1. The tagged femto-BS assigns the $i$ th idle RB to its femto user.

$p_{\mathrm{RB}}$ : The probability that the $i$ th idle RB being selected for data transmission by any of the other active CR femto-BS [32].

We show the detection result indicator of the $n$th RBs by $D_{n}(n \in\{1,2, \ldots, N\})$. If $D_{n}=1$, the $n$th RB is detected as idle RB, otherwise, $D_{n}=0$. The probability of one idle $\mathrm{RB}$ detected with no false alarm is $1-P_{f}$ and the probability for an occupied $R B$ detected as an idle $R B$ is $1-P_{d}$. In other words,

$$
\operatorname{Pr}\left(D_{n}=1\right)=\left\{\begin{array}{lc}
V_{0}=1-P_{f}, & \text { if } n \text {th RB isidle } \\
V_{1}=1-P_{d}, & \text { if } n \text {th RB is busy }
\end{array}\right.
$$

in which $P_{f}$ is the false alarm probability and can be obtained as follows [32-34]

$$
\begin{gathered}
P_{f}(\tau)=\mathcal{Q}\left(\sqrt{2 \eta+1} \mathcal{Q}^{-1}\left(P_{d}\right)+\sqrt{\tau f_{s}} \eta\right) \\
\mathcal{Q}(x)=\frac{1}{\sqrt{2 \pi}} \int_{x}^{\infty} \exp \left(\frac{-t^{2}}{2}\right) \mathrm{d} t \text { and } P_{d} \text { is the predefined de- }
\end{gathered}
$$

tection probability. $\tau$ is the spectrum sensing time, $f_{s}$ the sampling frequency, and $\eta$ the received interference power on an RB to each femto-BS.
Indeed, the probability that the $i$ th idle RB is detected with no false alarm by a CR femto-BS is $\operatorname{Pr}\left(D_{i}=1\right)=V_{0}$.

To obtain the probability that the $i$ th idle RB being selected for data transmission by a CR femto-BS $\left(p_{\mathrm{RB}}\right)$, first, we calculate the probability that the $i$ th idle RB is sensed and included in the $M_{s}$ idle RBs out of the $N_{s}$ sensed RBs in the sensing period $\left(T_{S}\right)$, and it is expressed as follows

$$
\begin{gathered}
\operatorname{Pr}\left(\text { the } i \text { th idle RB is sensed } \mid M_{s}\right) \\
=p_{\text {idle }}\left(M_{s}\right) \cdot \frac{\left(\begin{array}{c}
M-1 \\
M_{s}-1
\end{array}\right)}{\left(\begin{array}{c}
M \\
M_{s}
\end{array}\right)}
\end{gathered}
$$

where

$$
p_{\text {idle }}\left(M_{s}\right)=\frac{\left(\begin{array}{c}
M \\
M_{s}
\end{array}\right)\left(\begin{array}{c}
N-M \\
N_{s}-M_{s}
\end{array}\right)}{\left(\begin{array}{c}
N \\
N_{s}
\end{array}\right)}
$$

Conditioning on $M_{D}$ (see Table 1) and $M_{s}$, the probability that the $i$ th idle RB being detected as idle is obtained as follows

$$
\begin{aligned}
& \operatorname{Pr}\left(D_{i}=1 \mid M_{s}, M_{D}\right) \\
& =\operatorname{Pr}\left(D_{i}=1\right) \cdot \operatorname{Pr}\left(\sum_{n \neq i, n \in \Phi} D_{n}=M_{D}-1 \mid M_{s}\right) \\
& =V_{0} \times\left[\begin{array}{c}
\sum_{m_{\mathrm{D}}=\max \left\{1, M_{D}-\left(N_{s}-M_{s}\right)\right\}}^{\min \left\{M_{D}, M_{s}\right\}}\left[\left(\begin{array}{c}
M_{s}-1 \\
m_{\mathrm{ID}}-1
\end{array}\right)\left(V_{0}\right)^{m_{\mathrm{ID}}-1}\left(1-V_{0}\right)^{M_{s}-m_{\mathrm{ID}}}\right. \\
\left.\cdot\left(\begin{array}{c}
N_{s}-M_{s} \\
m_{\mathrm{OD}}
\end{array}\right) \cdot\left(V_{1}\right)^{m_{\mathrm{OD}}}\left(1-V_{1}\right)^{N_{s}-M_{s}-m_{\mathrm{OD}}}\right]
\end{array}\right]
\end{aligned}
$$

\section{Table 1 Symbols used in Section 4}

\begin{tabular}{ll}
\hline Symbols & Descriptions \\
\hline$N$ & Number of RBs \\
$M$ & Number of idle RBs \\
$N_{s}$ & Number of sensed RBs \\
$M_{s}$ & Number of idle RBs within the $N_{s}$ sensed RBs \\
$M_{D}$ & Number of RBs detected as idle within the $N_{s}$ sensed RBs \\
$m_{I D}$ & Number of idle RBs (out of the $M_{D}$ detected idle RBs) \\
& detected correctly. ( $m_{\mathrm{ID}} \in\left[\right.$ max $\left\{1, M_{s}-\left(N_{s}-M_{D}\right)\right\}$, \\
& min $\left.\left.\left\{M_{s}, M_{D}\right\}\right]\right)$ \\
$m_{\mathrm{OD}}$ & $\begin{array}{l}\text { Number of busy RBs (out of the } M_{D} \text { detected idle RBs) } \\
\left.\text { detected as idle. ( } m_{\mathrm{OD}}=M_{D}-m_{\mathrm{ID}}\right)\end{array}$ \\
\hline
\end{tabular}


in which $\Phi$ is the set of the detected RBs by a femto-BS (see Table 1 for the definitions of $m_{\mathrm{ID}}$ and $m_{\mathrm{OD}}$ ). Replacing $m_{\mathrm{OD}}$ with $M_{D}-m_{\mathrm{ID}}$, we have

$$
\begin{aligned}
& \operatorname{Pr}\left(D_{i}=1 \mid M_{s}, M_{D}\right) \\
& =\left[\begin{array}{c}
\sum_{m_{\mathrm{ID}}=}^{\min \left\{M_{D}, M_{s}\right\}}\left[1, M_{D}-\left(N_{s}-M_{s}\right)\right\} \\
\left.\cdot\left(\begin{array}{c}
N_{s}-M_{s} \\
M_{D}-m_{\mathrm{ID}}-1
\end{array}\right) \cdot\left(V_{1}\right)^{M_{D}-m_{\mathrm{ID}}}\left(1-V_{1}\right)^{N_{s}-M_{s}-M_{D}+m_{\mathrm{ID}}}\right]
\end{array}\right]
\end{aligned}
$$

Having the $M_{D}$ detected idle RBs (including the $i$ th idle $\mathrm{RB}$ ), the probability of a CR femto-BS accessing the $i$ th idle RB is equal to $\frac{1}{M_{D}}$. Thus, the probability that the $i$ th idle RB is selected for data transmission by any CR femto-BS (under imperfect sensing scenario) is obtained as follows

$$
\begin{aligned}
p_{\mathrm{RB} \mid M_{s}, M_{D}}= & \operatorname{Pr}\left(\text { the } i \text { th idle } \mathrm{RB} \text { is sensed } \mid M_{s}\right) \\
& \times \frac{1}{M_{D}} \times \operatorname{Pr}\left(D_{i}=1 \mid M_{s}, M_{D}\right)
\end{aligned}
$$

Finally,

$$
p_{\mathrm{RB}}=\sum_{M_{s}=\max \left\{1, N_{s}-(N-M)\right\}}^{\min \left\{N_{s}, M\right\}} \sum_{M_{D}=1}^{N_{s}} p_{\mathrm{RB} \mid M_{s}, M_{D}} .
$$

Case 2. The tagged femto-BS assigns the $i$ th busy RB (occupied by the macro network) to its femto user.

$p_{\mathrm{RB}}$ : The probability that the $i$ th busy $\mathrm{RB}$ being selected for data transmission by any of the other active CR femto-BS.

To obtain the probability of the $i$ th busy RB selected for data transmission by a femto-BS $\left(p_{\mathrm{RB}}\right)$, first, we calculate the probability that the $i$ th busy $R B$ is sensed and included in the $\left(N_{s}-M_{s}\right)$ busy RBs out of the $N_{s}$ sensed RBs in the sensing period, and it is expressed as follows

$$
\begin{gathered}
\operatorname{Pr}\left(\text { the } i \text { th busy RB is sensed } \mid\left(N_{s}-M_{s}\right)\right) \\
=\frac{\left(\begin{array}{c}
N-M-1 \\
N_{s}-M_{s}-1
\end{array}\right)\left(\begin{array}{c}
M \\
M_{s}
\end{array}\right)}{\left(\begin{array}{c}
N \\
N_{s}
\end{array}\right)} .
\end{gathered}
$$

Conditioning on $M_{D}$ and $\left(N_{s}-M_{s}\right)$, the probability that the $i$ th busy RB being detected as idle is obtained as follows

$$
\begin{aligned}
\operatorname{Pr}\left(D_{i}=1 \mid\left(N_{s}-M_{s}\right), M_{D}\right) \\
=\operatorname{Pr}\left(D_{i}=1\right) \cdot \operatorname{Pr}\left(\sum_{n \neq i, n \in \Phi} D_{n}=M_{D}-1 \mid\left(N_{s}-M_{s}\right)\right) \\
=V_{1} \times\left[\begin{array}{c}
\sum_{m_{\mathrm{ID}}=}^{\min \left\{M_{D}, M_{s}\right\}}\left\{1, M_{D}-\left(N_{s}-M_{s}\right)\right\} \\
\cdot\left(\begin{array}{c}
M_{s} \\
m_{\mathrm{ID}}-M_{s}-1 \\
m_{\mathrm{OD}}-1
\end{array}\right) \cdot\left(V_{0}\right)^{m_{\mathrm{ID}}}\left(1-V_{0}\right)^{M_{\mathrm{OD}}-1}\left(1-V_{\mathrm{ID}}\right.
\end{array}\right]
\end{aligned}
$$

in which $\Phi$ is the set of the detected RBs by a femto-BS. Again, replacing $m_{\mathrm{OD}}$ with $M_{D}-m_{\mathrm{ID}}$, we have

$$
\begin{aligned}
\operatorname{Pr}\left(D_{i}=1 \mid\left(N_{s}-M_{s}\right), M_{D}\right) \\
=\left[\begin{array}{c}
\left.\sum_{\mathrm{ID}}^{\min \left\{M_{D}, M_{s}\right\}}\left[\begin{array}{c}
M_{s}\left\{1, M_{D}-\left(N_{s}-M_{s}\right)\right\} \\
N_{s}-M_{s}-1 \\
M_{D}-m_{\mathrm{ID}}-1
\end{array}\right) \cdot\left(V_{1}\right)^{M_{D}-m_{\mathrm{ID}}}\left(1-V_{1}\right)^{N_{s}-M_{s}-M_{D}+m_{\mathrm{ID}}}\right]
\end{array}\right]
\end{aligned}
$$

Having the $M_{D}$ detected idle RBs (including the $i$ th busy RB), the probability of a CR femto-BS accessing the $i$ th busy RB is $\frac{1}{M_{D}}$. Thus, the probability that the $i$ th busy $\mathrm{RB}$ is selected for data transmission by any CR femto-BS (under imperfect sensing scenario) is obtained as follows

$$
\begin{aligned}
p_{\mathrm{RB} \mid\left(N_{s}-M_{s}\right), M_{D}}=\operatorname{Pr}\left(\text { the } i \text { th idle } \mathrm{RB} \text { is sensed } \mid\left(N_{s}-M_{s}\right)\right) \\
\times \frac{1}{M_{D}} \times \operatorname{Pr}\left(D_{i}=1 \mid\left(N_{s}-M_{s}, M_{D}\right) .\right.
\end{aligned}
$$

Finally,

$$
p_{\mathrm{RB}}=\sum_{M_{s}=\max \left\{1, N_{s}-(N-M)\right\}}^{\min \left\{N_{s}, M\right\}} \sum_{M_{D}=1}^{N_{s}} p_{\mathrm{RB} \mid\left(N_{s}-M_{s}\right), M_{D}} .
$$

\section{Simulation results and discussions}

Before we present the obtained results, a brief discussion on the spatial distribution of the BSs and the macro exclusion regions is conducted as follows.

Talking about the femto outage probability, each femto user suffers from two sources of interference, i.e., macro and femto networks. For the macro network, the aggregate interference results from all macro-BSs that use the same $\mathrm{RB}$ as the tagged femto user (i.e., we define a homogenous PPP with intensity $\lambda_{M}^{\prime}$ ). For the femto network, the aggregate interference results only from the other femto-BSs that (1) pick the same RB as the tagged 
femto, (2) are allowed to transmit in the current time slot, and (3) are not inside the macro users' exclusion regions. Hence, the interfering femto-BSs do not constitute a homogeneous point process anymore, and analytical characterization of interference and outage in this case is hard to characterize (the resulting point process is called Poisson hole process). In the analysis, to keep the modeling tractable, we ignored the possible correlation between the locations of the interfering femtoBSs and approximated the spatial distribution of them by a homogeneous PPP of intensity $\lambda_{F}^{\prime} p_{\mathrm{RB}} p_{\mathrm{tx}}$. Authors in $[10,35]$ use the same approximation approach, where its accuracy is also justified by simulation in [10]. Similar arguments and approximations were considered for the macro tier outage probability.

As interferences are experienced at receivers, we centered the macro exclusion regions around the macro users. The femto-BSs inside these areas may be able to detect the macro signals and cease their transmissions. The exclusion regions are usually chosen to be centered at the location of the macro-BSs not the macro receivers based on the argument that it is easier to detect the macro-BSs than the macro receivers especially if the receivers are passive like TV receivers. However, if the macro receivers (users) can be localized, e.g., based on pilot signals or transmitted acknowledgments, our obtained results directly apply and the exclusion regions around macro users can make sense. If the macro users cannot be localized, the exclusion regions have to be formed around the macro-BSs. This scenario can be evaluated with slight changes in the proposed model. It should be noted that the location detection of the macro users is outside the scope of this paper, however, many schemes have been already proposed. Measuring the power leakage of local oscillator is a possible way to detect the presence of the macro passive users (see $[10,36])$. The hidden node problem in CR systems which makes it difficult to detect the macro users can be also tackled, e.g., by adding a margin to the RB access detection threshold accounting for shadow fading and receiver location uncertainty for worst-case scenarios [30].

First, the accuracy of our analytical results for the downlink analysis in the proposed model is validated by simulations, as shown in Figures 4 and 5. The simulations which are built on Matlab platform are carried out to plot the curves of outage probability versus the SINR threshold for the tagged femto and macro user, as shown in Figures 4 and 5, respectively. The considered scenario is a two-tier network (exactly following the network model described in Sections 2 and 3) over an approximately $1 \times 1 \mathrm{~km}$ square with the locations of different classes of BSs as realizations of independent PPPs of given densities and the tagged users located at the center. To have an estimate of the outage probability at the

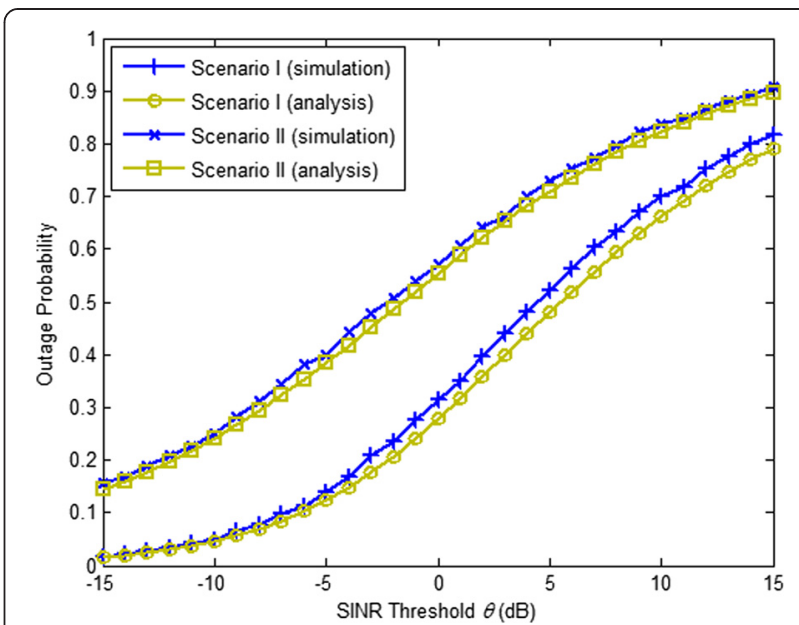

Figure 4 Outage probability of the tagged femto user. Outage probability of the tagged femto user (or any randomly chosen femto user) under perfect and imperfect sensing scenarios. The system parameters are set as $P_{F}=22 \mathrm{dBm}, P_{P}=42 \mathrm{dBm}, a=4$, $p_{\mathrm{RB}}=0.8, p_{\mathrm{tx}}=0.7, \mu=0.2, \mu_{p}=1, K=1, \lambda_{M}^{\prime}=12, \lambda_{F}=800$, and $\lambda_{F}^{\prime}=700$.

tagged users, the simulation results are averaged over both the spatial PPP (500 different positions) and fading distribution (300 realizations) and are conducted using the parameters mentioned in the figures' captions. Analytical curves are compared with the simulations under both perfect and imperfect sensing. It is observed that the simulation results closely match our analytical model, and the curves of analytical and simulation results match fairly well, which confirms our analysis. The plots exhibit slight discrepancies between analytical

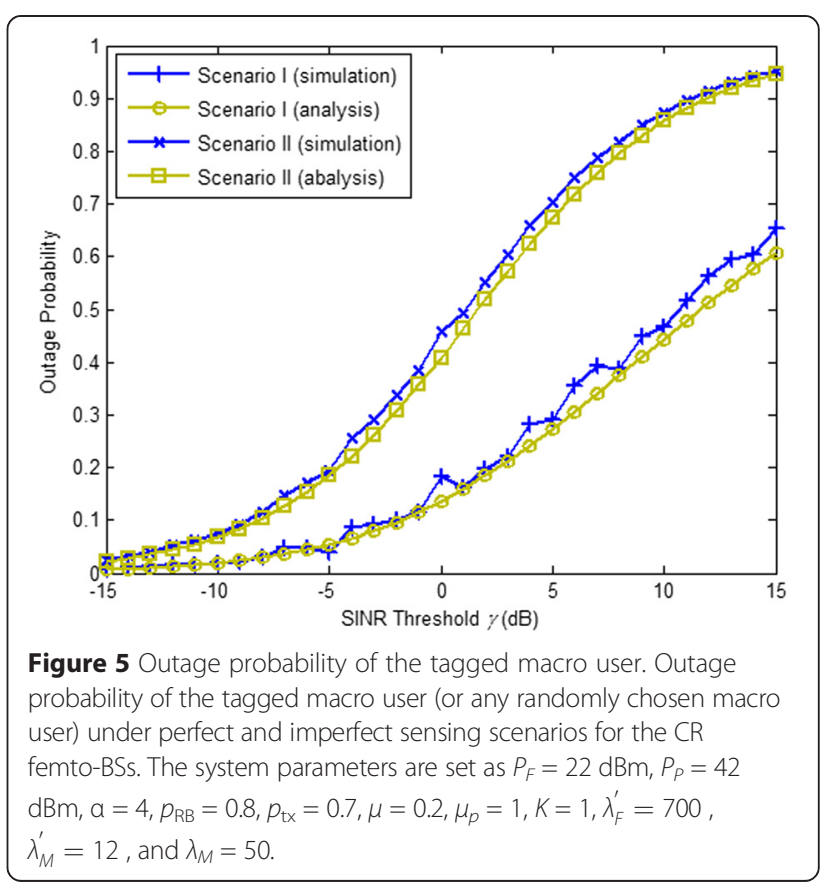


results and the corresponding simulation results which are mainly due to the independence assumption used in Section 3.

In Figure 6, the outage probability of the tagged femto user under perfect and imperfect spectrum sensing abilities for the CR femto-BSs is shown for different values of the target SINR $\theta$ on the horizontal axis. Our results show that the outage probability at the tagged femto receiver in the absence of a perfect spectrum sensing ability is considerably increased. In either the Scenario I, when all the femto-BSs employ perfect sensing to sense the RBs, or in Scenario II, Case 1, the tagged femto user does not experience any interference from the macroBSs owing to the correct detection at the tagged femtoBS or choice of idle RBs for data transmission (RBs not occupied by the macro network). In this case, the interference seen by the tagged femto user is only the aggregate interference from the other femto-BSs which are transmitting on the same idle RB as the tagged femto. Clearly, the lowest outage probability is achieved for this case (see the red curve). Now, let us consider the imperfect sensing scenario for the CR femto-BSs. Obviously, the tagged femto user is now subject to sensing error and therefore picking an occupied RB for its data transmission period. Under this condition, it may receive interference not only from the other femto-BSs which pick the same busy RB (due to the imperfect sensing) as the tagged femto but also from those macro-BSs communicating with their own users on the same RB as the tagged femto. Therefore, the tagged femto user experiences an interference larger than before and

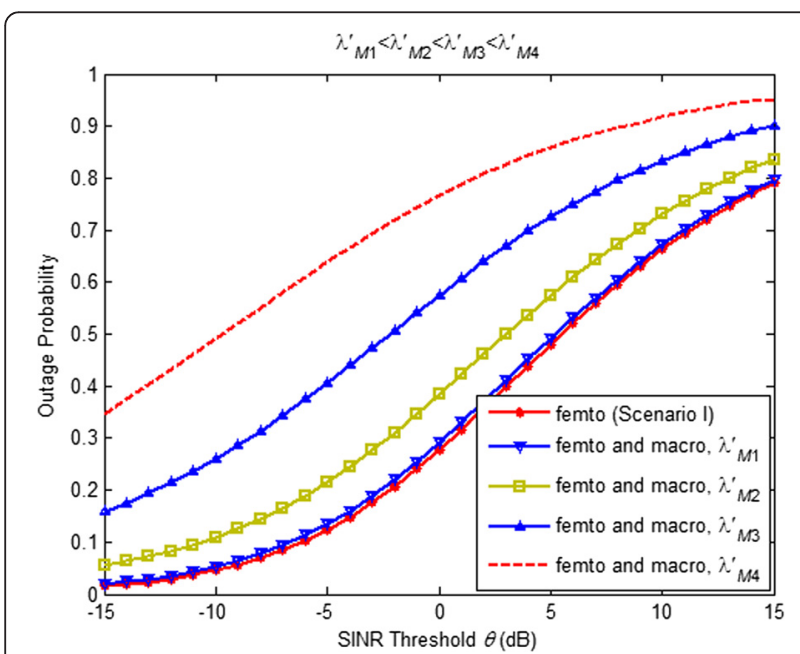

Figure 6 Outage probability of the tagged femto user with different values of $\lambda_{M}^{\prime}$. Outage probability of the tagged femto user (or any randomly chosen femto user) under perfect and imperfect sensing scenarios with different values of $\lambda_{M}^{\prime}$. The system parameters are set as $P_{F}=22 \mathrm{dBm}, P_{P}=42 \mathrm{dBm}, a=4, p_{\mathrm{RB}}=0.8, p_{\mathrm{tx}}=0.7, \mu=0.2$, $\mu_{P}=1, K=1, \lambda_{F}=800$, and $\lambda_{F}^{\prime}=700$. consequently a significant increase in the outage probability. Moreover, the tagged femto user will face an outage with a higher probability whenever it picks an RB (occupied RB) already used by a larger number of macro users (a larger $\lambda_{M}^{\prime}$ ) (see Figure 6).

Figure 7 depicts the outage probability of the tagged macro user for different values of the target SINR $\gamma$ and different situations. Considering the results obtained in Figure 6, here, we also investigate the effect of employing the two different sensing scenarios for the CR femto-BSs on the outage probability of the tagged macro user. In the case of perfect sensing, the tagged macro user does not experience any interference from the femto-BSs because only those RBs sensed to be idle (RBs not occupied by the macro network) are always chosen for data transmission by the femto network. In this case, the interference observed at the tagged macro user is only the aggregate interference received from those macro-BSs transmitting on the same RB as the tagged macro. Clearly, the lowest outage probability is obtained for this case (see the red curve). Now, the case of imperfect sensing of the CR femto-BS nodes is considered when the femto-BSs are subject to sensing error and therefore the possibility of transmitting on the RB occupied by the tagged macro. Under this condition, the tagged macro user may receive interference not only from the other macro-BSs communicating with their own users over the same RB (due to the lack of RBs) as the tagged macro but also from those femto-BSs which pick the same RB. Therefore, the tagged macro user experiences an interference larger than before and

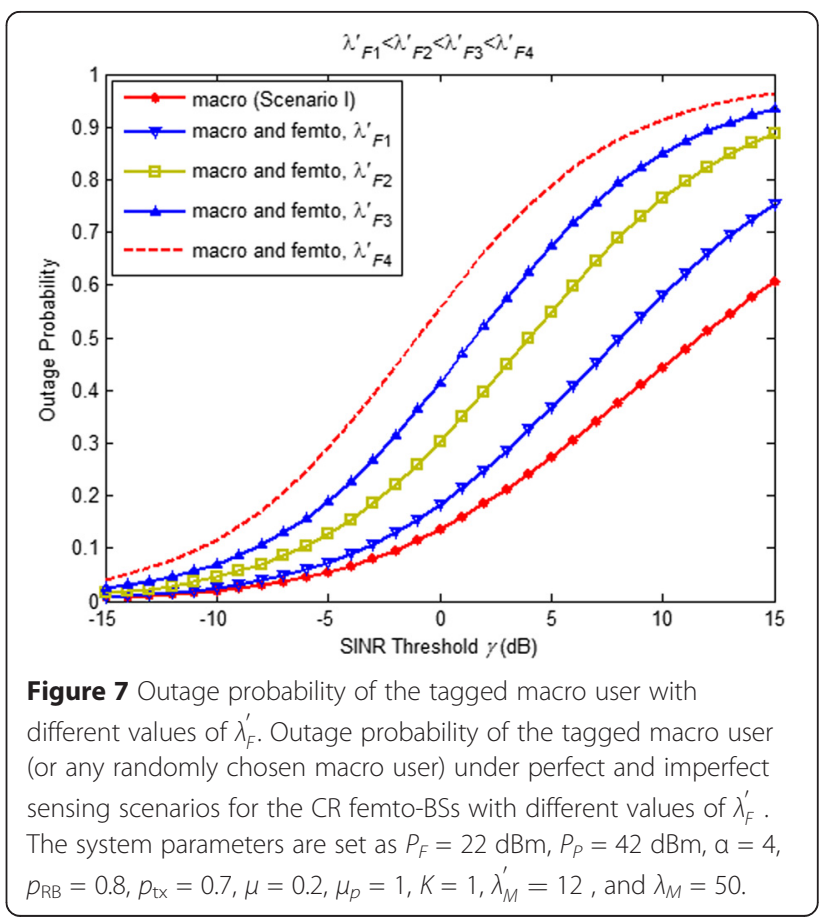


consequently a significant increase in the outage probability. Moreover, the tagged macro user will face an outage with a higher probability whenever its own RB is wrongly selected for data transmission by a larger number of femto-BSs (a larger $\lambda_{F}^{\prime}$ ).

Figure 8 illustrates the effect of $K$ (in $D=K r_{p}$ ) on the observed outage probability at the tagged macro user in the presence of both the macro and femto networks. Considering the previous explanations and Figure 3, let $\Phi_{F}^{\prime}$ include all the points (representing the femto-BSs) in $\Phi_{F}$ except the points inside the exclusion region $D$ of the tagged macro user. Since $\Phi_{F}^{\prime} \subset \Phi_{F}$, the potential aggregate interference at the tagged macro user, caused by the active (considering slotted ALOHA) CR femto-BSs, is less than that in the case with no $D$. Furthermore, as the exclusion region $D$ becomes larger (when $K=10$, for example), the probability of outage is significantly reduced. Indeed, the bigger the value of $D$, the closer the outage probability curve becomes to the black curve which represents the outage probability in the case when no overall interference from the femto network is observed at the tagged macro user due to the perfect sensing ability of the $\mathrm{CR}$ femto-BSs. However, the reduction in the outage probability can be less when the number of macro-BSs transmitting on the same RB as the tagged macro is larger (see Figure $8 \mathrm{~b}$ ).

For a commercial network, designers must find a way to achieve a lower probability of outage for a certain SINR as the minimum quality needed for a typical femto or macro user to experience an acceptable QoS. A common way to decrease the outage probability is to reduce the number of interfering BSs encountered at the users. This can be done through applying both the (a) and (b) conditions in the perfect sensing scenario (or both the (c) and (d) in the imperfect sensing scenario). As shown in Figure 9, under these constraints, the outage probability is significantly reduced at the tagged femto user (note that the number of interfering macro-BSs at the tagged femto user is considered equal for all curves in the figure). The goal is to see the effect of both $p_{\mathrm{RB}}$ and $p_{\mathrm{tx}}$ (these two parameters are employed at each CR femtoBS) on the outage probability of the tagged femto user. As can be seen, for the case when $p_{\mathrm{RB}}=1$ and $p_{\mathrm{tx}}=1$, outage occurs with higher probability. In other words, when all the existing femto-BSs (except those who are inside the macro users' exclusion regions) pick the same $\mathrm{RB}$ as the tagged femto $\left(p_{\mathrm{RB}}=1\right)$ and when they all have data to transmit in the current time slot $\left(p_{\mathrm{tx}}=1\right)$, the tagged femto user will experience the maximum value for the outage probability derived for different SINR targets. Clearly, a significant reduction in the outage probability is occurred for the smaller values of $p_{\mathrm{RB}}$ and $p_{\mathrm{tx}}$ (see Figure 9) (the smaller the values of $p_{\mathrm{RB}}$ and $p_{\mathrm{tx}}$, the closer the outage probability becomes to the outage probability in the case when the received interference at the tagged femto user is only the aggregate interference from the macro network). Indeed, this validates that many studies which do not consider these constraints over-estimate the interference encountered by a typical femto user.

It can be seen that even for high values of $p_{\mathrm{tx}}$, the outage probability is relatively less than that in the case where there is no constraint on the femto-BS's transmission schedule $\left(p_{\mathrm{tx}}=1\right)$. Also, it is obvious that in the presence of multiple RBs where each RB is picked with probability $p_{\mathrm{RB}}$, the outage probability is further decreased. Hence,

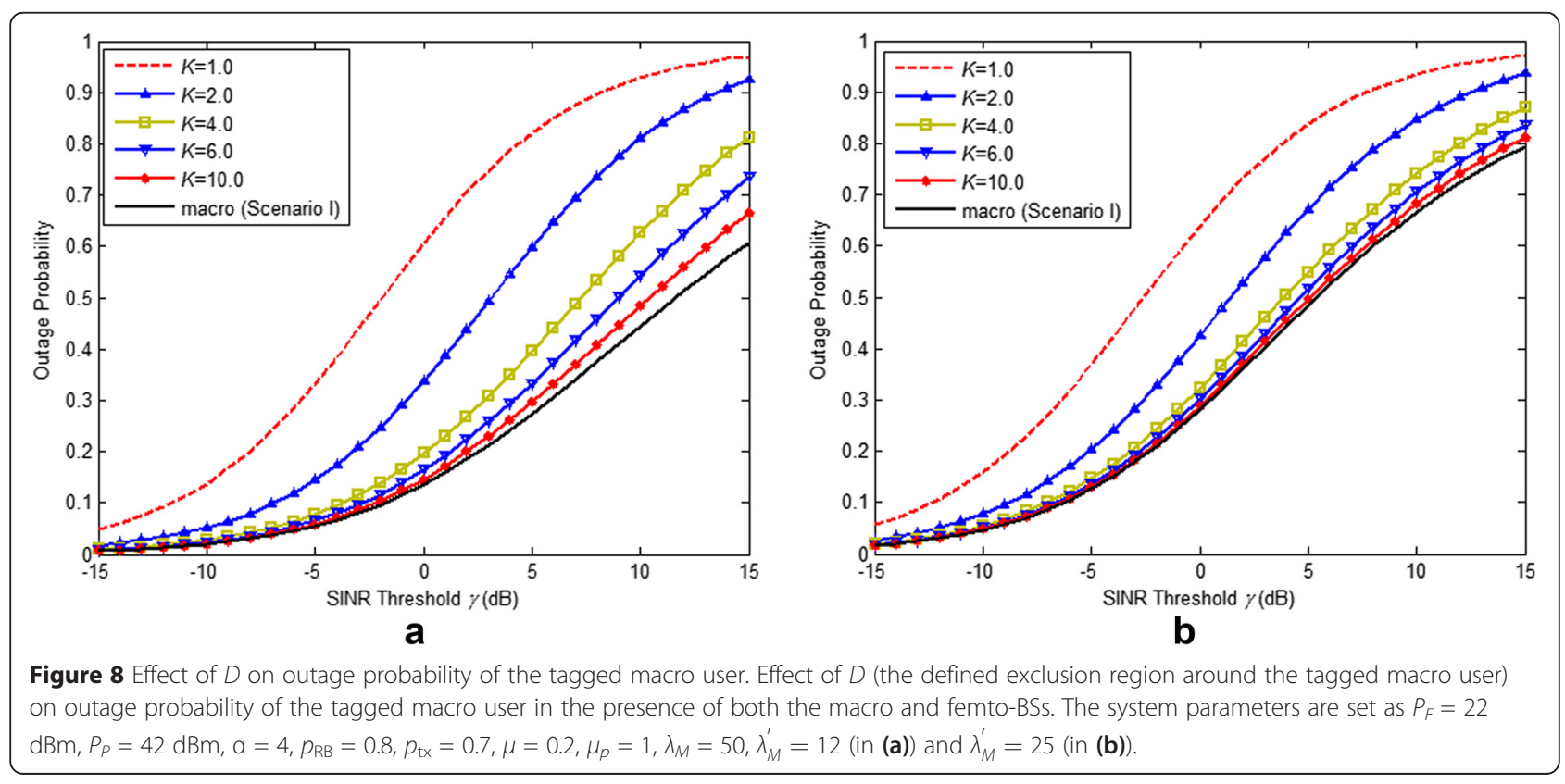




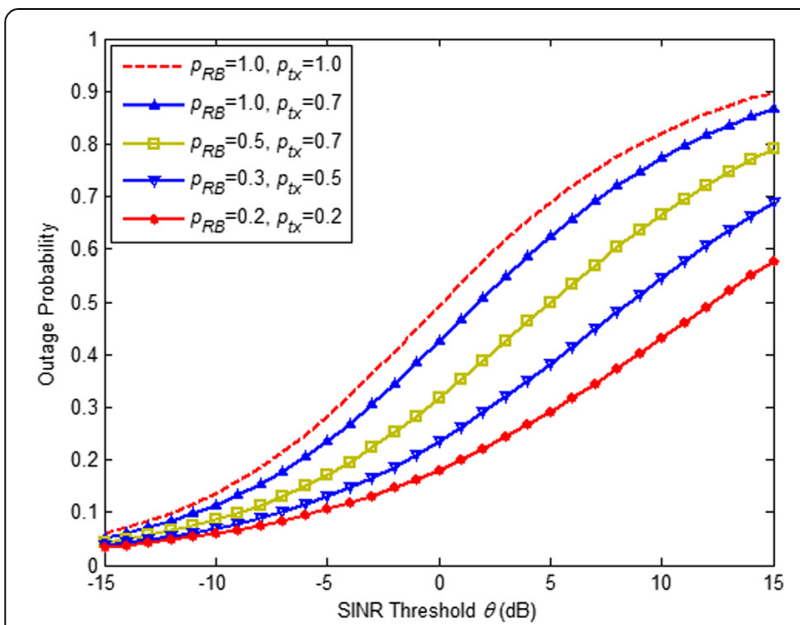

Figure 9 Effect of $p_{\mathrm{RB}}$ and $p_{\mathrm{tx}}$ on outage probability of the tagged femto user. Effect of $p_{\mathrm{RB}}$ and $p_{\mathrm{tx}}$ (the defined parameters for the $\mathrm{CR}$ femto-BSs) on outage probability of the tagged femto user in the presence of both the macro and femto-BSs. The system parameters are set as $P_{F}=22 \mathrm{dBm}, P_{P}=42 \mathrm{dBm}, a=4, \mu=0.2, \mu_{p}=1, K=1$, $\lambda_{F}=800, \lambda_{F}^{\prime}=700$, and $\lambda_{M}^{\prime}=12$.

any practical heterogeneous network designed to satisfy both of the mentioned conditions can reap the benefit of opportunistic exploitation of spectrum, while possibly causing little or no harmful interference. The same story exists when the outage probability of the tagged macro user is investigated. Figure 10 shows the effect of $p_{\mathrm{RB}}$ and $p_{\mathrm{tx}}$ (parameters which are related to the CR femto-BSs) on the outage probability of the tagged macro user. For

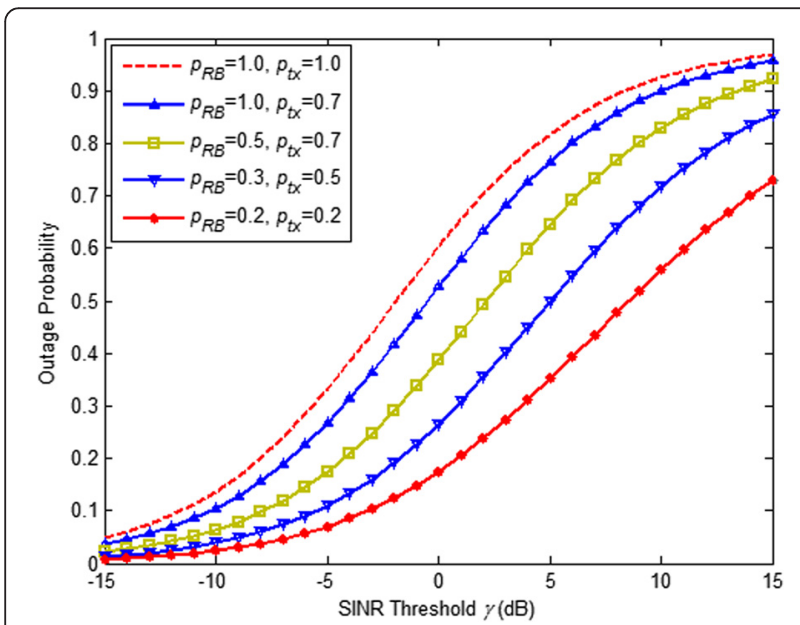

Figure 10 Effect of $p_{\mathrm{RB}}$ and $p_{\mathrm{tx}}$ on outage probability of the tagged macro user. Effect of $p_{\mathrm{RB}}$ and $p_{\mathrm{tx}}$ (the defined parameters for the CR femto-BSs) on outage probability of the tagged macro user in the presence of both the macro and femto-BSs. The system parameters are set as $P_{F}=22 \mathrm{dBm}, P_{P}=42 \mathrm{dBm}, \mathrm{a}=4, \mu=0.2, \mu_{p}=1, K=1$, $\lambda_{F}^{\prime}=700, \lambda_{M}=50$, and $\lambda_{M}^{\prime}=12$. instance, when all the active femto-BSs select the same $\mathrm{RB}$ as the tagged macro $\left(p_{\mathrm{RB}}=1\right)$ and when they all have data to transmit (on this busy RB) in the current time slot $\left(p_{\mathrm{tx}}=1\right)$, the tagged macro user will experience the maximum value for the outage probability. It should be noted that the number of interfering macro-BSs at the tagged macro is considered equal for all curves in this figure.

In Figures 11 and 12, we analyze the performance of the authorized links (femto links) in terms of throughput (achievable with a simple ARQ scheme with error-free feedback) under both the perfect and imperfect sensing scenarios. Considering the slotted ALOHA scheme, we define the following terms $[37,38]$,

Probabilistic link throughput $(\tau)$ of a femto user:

(i) in the half-duplex (HD) communication scenario: it is defined to be the success probability of a femto user (i.e., $1-p_{\mathrm{OF}}$ ) multiplied by the probability that the corresponding femto-BS actually transmits over a specific RB (i.e., $p_{\mathrm{RB}} p_{\mathrm{tx}}$ ), and the probability that the femto receiver actually receives over that $\mathrm{RB}$ (i.e., $1-p_{\mathrm{RB}} p_{\mathrm{tx}}$ )

(ii)in the full-duplex (FD) communication scenario: it is defined to be the success probability of a femto user (i.e., $1-p_{\mathrm{OF}}$ ) multiplied by the probability that the corresponding femto-BS actually transmits over a specific RB (i.e., $p_{\mathrm{RB}} p_{\mathrm{tx}}$ ).

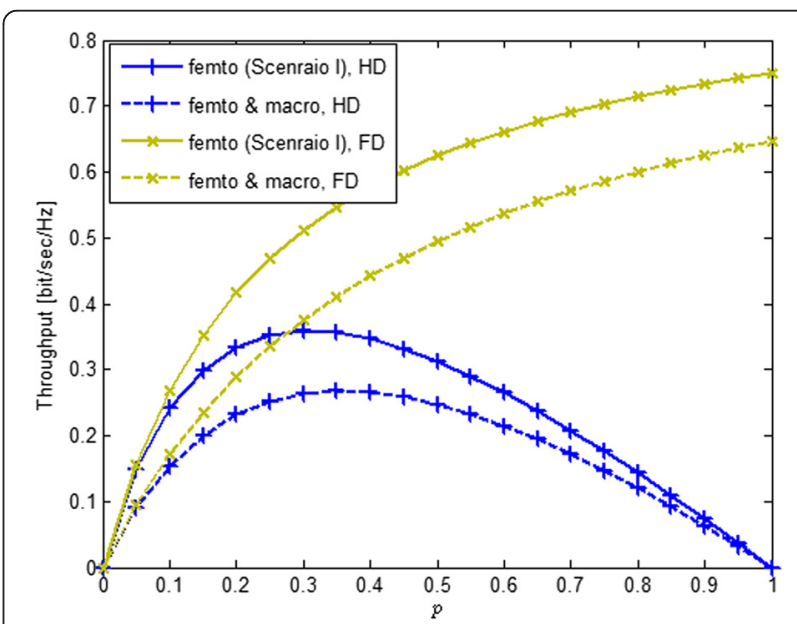

Figure 11 The femto link throughput of half- and full-duplex systems as a function of the transmission probability. The femto link throughput of half- and full-duplex systems under perfect and imperfect sensing scenarios as a function of the transmission probability over a specific RB (p). The system parameters are set as $P_{F}=22 \mathrm{dBm}, P_{P}=42 \mathrm{dBm}, a=4, \mu=0.2, \mu_{P}=1, K=1, \lambda_{M}^{\prime}=12$, $\lambda_{F}=800$ and $\lambda_{F}^{\prime}=700$, and $\theta=11 \mathrm{~dB}$. 


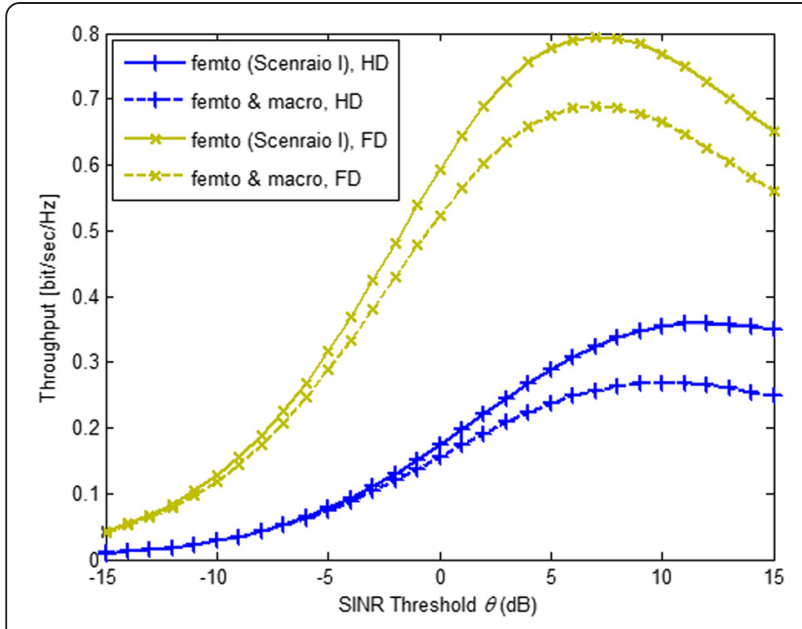

Figure 12 The femto link throughput of half and full-duplex systems as a function of the target SINR. The femto link throughput of half and full-duplex systems under perfect and imperfect sensing scenarios as a function of the target SINR. The system parameters are set as

$P_{F}=22 \mathrm{dBm}, P_{p}=42 \mathrm{dBm}, \mathrm{a}=4, \mu=0.2, \mu_{p}=1, K=1, \lambda_{M}^{\prime}=12$,

$\lambda_{F}=800$, and $\lambda_{F}^{\prime}=700$; and for each curve, the optimal value of $p$ obtained in Figure 11 is applied.

\section{Femto link throughput (T):}

The femto link throughput is defined as the product of the probabilistic link throughput $(\tau)$ and the rate of transmission, i.e., $T=\tau \log (1+\theta)$. Therefore, the femto link throughput for the half and full-duplex cases is written as follows

$$
\begin{array}{ccc}
T^{(\text {half })} & = & p(1-p)\left(1-p_{\mathrm{OF}}\right) \log (1+\theta) \\
T^{(\mathrm{full})} & = & p\left(1-p_{\mathrm{OF}}\right) \log (1+\theta)
\end{array}
$$

in which $p=p_{\mathrm{RB}} p_{\mathrm{tx}}$.

In Figure 11, the performance of half- and full-duplex systems is presented for the femto users. More specifically, the link throughput of any typical femto user (e.g., the link between the tagged femto user and its corresponding femto-BS) under perfect and imperfect spectrum sensing abilities for the CR femto-BSs is shown as a function of the transmission probability over a specific $\mathrm{RB}$ (i.e., $p=p_{\mathrm{RB}} p_{\mathrm{tx}}$ ). It can be seen that the throughput achieved by the FD system is significantly higher, particularly when $p$ is high. Regarding the performance of the HD system, for both the perfect and imperfect sensing cases, there is a unique optimal $p$ which achieves the maximum throughput ( $p=0.3$ for the perfect and $p=0.35$ for the imperfect sensing scenario). However, for high $p$, both throughput curves converge to zero due to over many transmissions and interferences on the RB. Obviously, for both the half- and full-duplex communications, a higher per-link throughput is achieved when the CR femto-BSs employ perfect sensing.
In Figure 12, the performance of half- and full-duplex systems is presented for femto users. More specifically, the link throughput of any typical femto user (e.g., the link between the tagged femto user and its corresponding femto-BS) under perfect and imperfect spectrum sensing abilities for the CR femto-BSs is shown as a function of the target $\operatorname{SINR} \theta$. It can be seen that the per-link throughput achieved by the FD system, for both the perfect and imperfect sensing scenarios, is significantly higher than the HD one. As it is seen, the link throughput curves are concave and there is an optimal point in each curve. With a high-target SINR, we can transmit the user data with high spectral efficiency; however, the outage probability of this transmission is high, too. In contrast, with a low-target SINR, we can send many packets that include little information. In other words, a high reliable transmission can be experienced at low-target SINRs, while the minimum requirements for the transmission rate cannot be met.

\section{Conclusions}

In this paper, utilizing the spatial Poisson point process (PPP) theory, we presented a tractable model to derive the outage probability of a typical femto and macro user in a two-tier heterogeneous network which provides insight into system design guidelines. In other words, for the case of the node locations modeled by a PPP and the desired and interfering channels are subject to Rayleigh fading, we demonstrated the use of the cognitive radio (CR)-based framework to evaluate the outage probability at any arbitrary user. Exact closed-form expressions were obtained as a result. In addition, we observed that in the downlink analysis, the outage probability is a function of the network topology and several important system design parameters such as SINR target, exclusion regions, MAC mechanisms such as ALOHA $\left(p_{\mathrm{tx}}\right)$, and the resource block $(\mathrm{RB})$ selection constraint $\left(p_{\mathrm{RB}}\right)$ which is controlled by the spectrum sensing measurements.

\section{Appendix I}

DERIVATION OF $M(\theta, \alpha)$

Proof: From the expression of $M(\theta, \alpha)$ in (11), we have

$$
M(\theta, \alpha)=\int_{0}^{\infty}\left[(g)^{\frac{d}{\alpha}}\left(\Gamma\left(-\frac{d}{\alpha}, \mu \theta g\right)-\Gamma\left(-\frac{d}{\alpha}\right)\right)\right] \mu e^{-\mu g} \mathrm{~d} g
$$

We know that $\Gamma(a, x)+\gamma(a, x)=\Gamma(a)$ and $\gamma(a, x)=x^{a}$ $\Gamma(a) e^{-x} \sum_{k=0}^{\infty} \frac{x^{k}}{\Gamma(a+k+1)}$, therefore, the above equation is simplified as follows 


$$
\begin{gathered}
=-\Gamma\left(-\frac{d}{\alpha}\right) \mu(\mu \theta)^{-\frac{d}{\alpha}} \int_{0}^{\infty} e^{-g(\mu+\mu \theta)}\left[\sum_{k=0}^{\infty} \frac{(\mu \theta)^{k} g^{k}}{\Gamma\left(-\frac{d}{\alpha}+k+1\right)}\right] \mathrm{d} g \\
=-\Gamma\left(-\frac{d}{\alpha}\right) \mu(\mu \theta)^{-\frac{d}{\alpha}} \int_{0}^{\infty} e^{-g(\mu+\mu \theta)} \\
\times\left[\frac{(\mu \theta)^{0} g^{0}}{\Gamma\left(1-\frac{d}{\alpha}\right)}+\frac{(\mu \theta)^{1} g^{1}}{\Gamma\left(2-\frac{d}{\alpha}\right)}+\frac{(\mu \theta)^{2} g^{2}}{\Gamma\left(3-\frac{d}{\alpha}\right)}+\ldots\right] \mathrm{d} g \\
=-\Gamma\left(-\frac{d}{\alpha}\right) \mu(\mu \theta)^{-\frac{d}{\alpha}}\left[\int_{0}^{\infty} \frac{(\mu \theta)^{0}(g)^{(1)-1} e^{-g(\mu+\mu \theta)}}{\Gamma\left(1-\frac{d}{\alpha}\right)} \mathrm{d} g\right. \\
\left.+\int_{0}^{\infty} \frac{(\mu \theta)^{1}(g)^{(2)-1} e^{-g(\mu+\mu \theta)}}{\Gamma\left(2-\frac{d}{\alpha}\right)} \mathrm{d} g+\ldots\right] \\
=-\Gamma\left(-\frac{d}{\alpha}\right) \mu(\mu \theta)^{-\frac{d}{\alpha}}\left[\frac{(\mu \theta)^{0}}{\Gamma\left(1-\frac{d}{\alpha}\right)(\mu+\mu \theta)} \Gamma(1)\right. \\
\left.+\frac{(\mu \theta)^{1}}{\Gamma\left(2-\frac{d}{\alpha}\right)(\mu+\mu \theta)^{2}} \Gamma(2)+\ldots\right]
\end{gathered}
$$

Hence, we have

$$
\begin{aligned}
M(\theta, \alpha)= & \left(-\Gamma\left(-\frac{d}{\alpha}\right) \mu(\mu \theta)^{-\frac{d}{\alpha}}\right) \\
& \times\left[\sum_{k=0}^{\infty} \frac{(\mu \theta)^{k}}{\Gamma\left(k+1-\frac{d}{\alpha}\right)(\mu+\mu \theta)^{k+1}} \Gamma(1+k)\right]
\end{aligned}
$$

\section{Competing interests}

The authors declare that they have no competing interests.

\section{Received: 1 September 2014 Accepted: 16 April 2015}

Published online: 22 May 2015

\section{References}

1. J Andrews, H Claussen, M Dohler, S Rangan, M Reed, Femtocells: past, present, and future. IEEE J Sel Areas Comm 30(3), 497-508 (2012)

2. SCheng, $\mathrm{S}$ Lien, $\mathrm{F} \mathrm{Hu}, \mathrm{K}$ Chen, On exploiting cognitive radio to mitigate interference in macro/femto heterogeneous networks. IEEE Wireless Comm Mag 18, 40-47 (2011)

3. H ElSawy, E Hossain, M Haenggi, Stochastic geometry for modeling, analysis, and design of multi-tier and cognitive cellular wireless networks: a survey. IEEE Comm Surveys Tutorials 15(3), 996-1019 (2013)

4. K Gilhousen, I Jacobs, R Padovani, AJ Viterbi, L Weaver, C Wheatley, On the capacity of a cellular CDMA system. IEEE Trans Veh Tech 40(2), 303-312 (1991)

5. J Xu, J Zhang, JG Andrews, On the accuracy of the Wyner model in cellular networks. IEEE Trans Wireless Comm 10(9), 3098-3109 (2011)

6. JG Andrews, F Baccelli, RK Ganti, A tractable approach to coverage and rate in cellular networks. IEEE Trans Comm 59(11), 3122-3134 (2011)

7. SAR Zaidi, DC McLernon, M Ghogho, Outage probability analysis of cognitive radio networks under self-coexistence constraint, 44th Annual Conference on Information Sciences and Systems (CISS), 2010, pp. 1-6-17-19

8. RK Ganti, M Haenggi, Interference and outage in clustered wireless ad hoc networks. IEEE Trans Information Theory 55, 4067-4086 (2009)
9. A Hasan, JG Andrews, The guard zone in wireless ad hoc networks. IEEE Trans Wireless Comm 4(3), 897-906 (2007)

10. C-H Lee, M Haenggi, Interference and outage in Poisson cognitive networks. IEEE Trans Wireless Comm 11, 1392-1401 (2012)

11. V Chandrasekhar, JG Andrews, M Tarik, S Zukang, G Alan, Power control in two-tier femtocell networks. IEEE Trans Wireless Comm 8(8), 4316-4328 (2009)

12. HS Jo, C Mun, J Moon, JG Yook, Interference mitigation using uplink power control for two-tier femtocell networks. IEEE Trans Wireless Comm 8(10), 4906-4910 (2009)

13. V Chandrasekhar, JG Andrews, Spectrum allocation in tiered cellular networks. IEEE Trans Comm 57(10), 3059-3068 (2009)

14. D Cao, S Zhou, Z Niu, Improving the energy efficiency of two-tier heterogeneous cellular networks through partial spectrum reuse. IEEE Trans Wireless Comm 12(8), 4129-4141 (2013)

15. J Xiang, Y Zhang, T Skeie, L Xie, Downlink spectrum sharing for cognitive radio femtocell networks. IEEE Sys J 4(4), 524-534 (2010)

16. Y Shi, YT Hou, H Zhou, SF Midkiff, Distributed cross-layer optimization for cognitive radio networks. IEEE Trans Veh Tech 59(8), 4058-4069 (2010)

17. H Dhillon, R Ganti, F Baccelli, J Andrews, Modeling and analysis of K-Tier downlink heterogeneous cellular networks. IEEE J Selected Areas Comm 30(3), 550-560 (2012)

18. H Jo, Y Sang, P Xia, J Andrews, Outage Probability for Heterogeneous Cellular Networks with Biased Cell Association (Proc, IEEE GlobeCom, 2011)

19. T Novlan, R Ganti, A Ghosh, J Andrews, Analytical evaluation of fractional frequency reuse for OFDMA cellular networks. IEEE Trans Wireless Comm 10(12), 4294-4305 (2011)

20. T Novlan, R Ganti, A Ghosh, J Andrews, Analytical evaluation of fractional frequency reuse for heterogeneous cellular networks. IEEE Trans Comm 60(7), 2029-2039 (2012)

21. FH Panahi, T Ohtsuki, Stochastic Geometry Based Analytical Modeling of Cognitive Heterogeneous Cellular Networks, IEEE International Conf. on Comm. (ICC), 2014, pp. 5281-5286

22. H ElSawy, E Hossain, Two-tier HetNets with cognitive femtocells: downlink performance modeling and analysis in a multi-channel environment. IEEE Trans Mobile Computing 13(3), 649-663 (2014)

23. H ElSawy, E Hossain, DI Kim, HetNets with Cognitive Small Cells: User Offloading and Distributed Channel Allocation Techniques, IEEE Communications Magazine, Special Issue on Heterogeneous and Small Cell Networks (HetSNets), vol. , 2013

24. H ElSawy, E Hossain, Channel Assignment and Opportunistic Spectrum Access in Two-Tier Cellular Networks with Cognitive Small Cells, in Proc. IEEE Global Communications Conference (Globecom), 2013

25. H ElSawy, E Hossain, On Cognitive Small Cells in Two-Tier Heterogeneous Networks, Workshop on Spatial Stochastic Models for Wireless Networks (SpaSWiN), in conjunction with 11th Intl. Symposium on Modeling and Optimization in Mobile, Ad Hoc, and Wireless Networks (WiOpt), 2013

26. M Haenggi, RK Gant, Interference in Large Wireless Networks. Foundations and Trends in Networking, NOW Publisher, 2008. vol. 3, no. 2

27. IEEE Instrumentation, M Society, IEEE 1588 Standard for a Precision Clock Synchronization Protocol for Networked Measurement and Control Systems, in IEEE Std 1588, 2008

28. Q Wu, G Ding, J Wang, Y-D Yao, Spatial-temporal opportunity detection in spectrum-heterogeneous cognitive radio networks: two dimensional sensing. IEEE Trans Wireless Comm 12(2), 516-526 (2013)

29. Y Chen, Q Zhao, A Swami, Joint design and separation principle for opportunistic spectrum access in the presence of sensing errors. IEEE Trans Inf Theory 54(5), 2053-2071 (2008)

30. F Paisana, N Marchetti, LA Dasilva, Radar, TV and Cellular Bands: Which Spectrum Access Techniques for which Bands? IEEE Comm. Surveys \& Tutorials, vol.16, no.3, pp.1193-1220, Third Quarter, 2014

31. Q Chen, M Motani, W-C Wong, Y-C Liang, Opportunistic Spectrum Access Protocol for Cognitive Radio Networks, in Proc. IEEE International Conference on Comm. (ICC), 2011, pp. 1-6

32. X Li, H Liu, S Roy, J Zhang, P Zhang, C Ghosh, Throughput Analysis for a Multi-User, Multi-Channel ALOHA Cognitive Radio System. IEEE Trans. on Wireless Comm. 11(11), 3900-3909 (2012)

33. Y-C Liang, Y Zeng, E Peh, AT Hoang, Sensing-throughput tradeoff for cognitive radio networks. IEEE Trans Wireless Comm 7(4), 1326-1337 (2008)

34. L Luo, C Ghosh, S Roy, Joint Optimization of Spectrum Sensing for Cognitive Radio Networks, in Proc. IEEE Global Telecommunications Conference (GLOBECOM), 2010, pp. 1-5 
35. L Wang, $V$ Fodor, On the gain of primary exclusion region and vertical cooperation in spectrum sharing wireless networks. IEEE Trans Veh Tech 61(8), 3746-3758 (2012)

36. T Yucek, H Arslan, A Survey of Spectrum Sensing Algorithms for Cognitive Radio Applications, IEEE Comm. Surveys \& Tutorials, vol. 11, no. 1, pp. 116-130, First Quarter, 2009

37. M Haenggi, Outage, local throughput, and capacity of random wireless networks. IEEE Trans Wireless Comm 8(8), 4350-4359 (2009)

38. J-M Dricot, G Ferrari, A Panahandeh, H Fr, D Ph De, Probabilistic Coexistence and Throughput of Cognitive Dual-Polarized Networks. EURASIP Journal on Wireless Comm. and Networking, 2010. 387625

Submit your manuscript to a SpringerOpen ${ }^{\odot}$ journal and benefit from:

- Convenient online submission

- Rigorous peer review

- Immediate publication on acceptance

- Open access: articles freely available online

- High visibility within the field

- Retaining the copyright to your article

Submit your next manuscript at $\gg$ springeropen.com 